\title{
Contextual metrics A mathematical definition for a comprehensive approach of geographical distances
}

\author{
Benoît R. Kloeckner ${ }^{1}$, Alain L'Hostis ${ }^{2}$, and Thomas Richard ${ }^{1}$ \\ ${ }^{1}$ Université Paris-Est, Laboratoire d'Analyse et de Mathématiques \\ Appliquées (UMR 8050), UPEM, UPEC, CNRS, F-94010, Créteil, \\ France \\ ${ }^{2}$ LVMT, Univ Gustave Eiffel, IFSTTAR, Ecole des Ponts, F-77454 \\ Marne-la-Vallée, France
}

September 24, 2020

\begin{abstract}
Our goal is to establish a mathematical framework for the description of geographical distance in a comprehensive way. Geographical distance always refer to potential or realized movement between places, and these displacements obey the least effort rule. While this optimization of effort is well known to imply the Triangle inequality in many situation, breaks in movement generate a paradox: effort optimization, taking into account the need to rest, results in apparent violations of the Triangle Inequality. In order to solve this issue, we introduce contextual metrics that consider space but also any contextual information relevant to travel, such as resources used for moving. Our approach permits to build a subjective space where distances are affected by the characteristics of the individual on the move. Contextual metrics frame the optimization problem in a space enriched by the context that the traveler has to take into account, making apparent that the violation of the Triangle Inequality in case of break was only an artefact of a model lacking crucial information. The range of geographical situations that can be modelled with this framework underline the level of generalization that can be expected from this approach.
\end{abstract}




\section{Introduction}

Distance is a central concept for both geography and geometry. In geography, our focus, distance is generated by the necessary separation of geographic entities: all geographic objects can't occupy the same place (Isnard et al. 1981). If the "central problem of geography is to place interacting objects as near to each other as possible when the definition of distance is chosen which minimizes movement" (Bunge 1962, p. 200), then distance definition is a critical task for the geographer. Three modes of management of geographic distances, at least since the neolithic period, have been observed: co-presence, mobility and telecommunication (Lévy 2009). Hence transport is essential for understanding geographical distances.

Tobler's First Law of geography (TFL), "everything is related to everything else, but near things are more related than distant things" (Tobler 1970), has slowly made its way in geography (Sui 2004; Miller 2004b) and is now recognized as a "near universally respected reflection" in the discipline (Anselin and Li 2020, p. 13). It has been discussed how much relevant it remained in a shrinking world, where places are brought closer by new means of transportation and by increasingly efficient telecommunication systems, but its relevance seems to have rather increased - reduced distances where indeed followed by increased interactions (Kirsch 1995; Ahmed and Miller 2007). To what extent does TFL conveys a definition of geographical distances? If we are to understand how different locations are related, a key point according to TFL is to understand how near or far they are from each other. But even when focusing on mobility, near and far should not be understood in a purely physical, as the crow flies, sense: two locations linked by an expressway should entertain a stronger relation with each other than two locations nearer in physical space, but separated by rough ground. It is thus the usual understanding of TFL that nearness is to be measured in ease of travel. One small additional step leads us to define distances by the difficulty in relating two locations. TFL applied to mobility is then reduced to the statement that we do more what we can easily do; whether any potential movement will actually take place or not is guided by the expected ratio of benefit over cost; and the way in which the movement will occur (choice of path, of transport mean) will be guided by attempting minimization of the cost (Zipf 1949; Warntz 1967; Wu and Miller 2001; Geertman and Eck 1995). Therefore, in order to understand movements in a geographical space it is necessary to consider more than the physical lengths, and by distance we will mean a disutility of arbitrary nature associated to movement. Moreover, in order to gain deeper understanding of movement, one has to take into account every element that influences the incurred disutility, as they may affect whether and how the movement will take 
place.

Our intention is to establish a mathematical framework suitable for the description of geographical distances related to movement in space and produced by transport means, measured in arbitrary units and taking into account any relevant context.

The geography of distances has been the focus of many geographical and several spatial economy contributions. A related problem is the cartography of timedistances (Shepard 1962; Marchand 1973; Kruskal and Wish 1978; G.M. Hyman and Mayhew 2004; Axhausen et al. 2008; Shimizu and Inoue 2009; Ficzere, Ultmann, and Török 2014; Dusek and Szalkai 2017). Contributions in this field develop practical solutions that minimize or control the stress of the cartographic representation or use differential geometry (Angel and Geoffrey Hyman 1976), but do not introduce new mathematical definitions of distances. In transport studies and in the literature on the measurement of accessibility, we find references to distances measurement that involve routing (Ritsema van Eck and de Jong 1999; Kim and Kwan 2003; Delafontaine, Neutens, and Weghe 2012), with time dependant graphs (Chalmet, Francis, and Saunders 1982), or including transit schedules routing (O'Sullivan, Morrison, and Shearer 2000; Lozano and Storchi 2001; Nuzzolo, Russo, and Crisalli 2001; MüllerHannemann et al. 2007; Lei and Church 2010; Ma and Lebacque 2013; W. Zeng et al. 2014; Bast et al. 2016). Nevertheless, this literature focuses on the methods and algorithms aimed at generating high quality distance datasets and associated maps, but do not elaborate on the mathematical nature of these distances. Most of these works take inspiration from the time geography framework (Hägerstrand 1970). In the time geography stream we find developments in geometry focusing on the shapes needed in this framework, with path, prisms (Kuijpers, Miller, and Othman 2017), bundles and other spatial objects (Miller 2005), but, again, not on the mathematics of distance.

The set of approaches the closest to our intention have concentrated on the development of a mathematical distance framework suitable for the computation of transport costs, in the context of spatial economy (Smith 1989; Huriot, Smith, and Thisse 1989). These contributions have provided a theoretical foundation for the development of geographical distance measurement on networks (Dean 1997; Ahmed and Miller 2007). Our own focus, on the geometry of geographical distances, differs from Smith (1989) and Huriot, Smith, and Thisse (1989) in the sense that it cares more for geographical and cartographic issues while also considering spatial economy costs.

An additional important feature of the current work, and actually its initial motivation, is theoretical and concerns the Triangle Inequality. It has often been considered irrelevant to certain geographical distances, but we shall show that the triangular 
inequality holds in one form or another universally, in a very wide framework, since it follows logically from the minimization process. We close a critic of the critics of the Triangular Inequality opened in L'Hostis (2016), by showing that last remaining case where it seemed not to hold is in fact a case where distances are ill-defined, precisely because they depend on a context.

A context will be information to be added to the location and that will influence the available choices of movement from this location and their costs. Examples of contexts are numerous: available budget, fuel gauge level, accumulated fatigue, date and time of day, etc. Instead of considering movement solely into physical space, we will recast it into an enriched space whose elements are called states and compound location and context; in particular, this enables us to express distance to a location not only in term of the starting location, but also in term of the context in which the traveler finds herself or himself.

In appendix A, we shall develop a more detailed framework based on elementary trips that are composed into paths in the enriched space. The important point is that each elementary trip can change the context (spend some money, or fuel, add some fatigue, shift the time of day, etc.), thus potentially influencing all subsequent parts of the journey.

The two facets of the enriched space, geometry and context, do not play symmetric roles. The geometry is immutable, at least at the timescale involved in the movement, while the context will usually evolve as the movement proceeds. The geometry only takes into account places (starting location and target location), while context denotes all other movement-related circumstances.

Our framework being flexible, contexts can also be used to model differences between travelers (e.g. differences in wealth or in preferences, i.e. differences that are intrinsic to the travelers and not related to their locations, and thus are ignored in traditional models of geographic distances). Beyond a purely physical approach of space, what we model is closer to space as it is experienced by the traveler: different travelers, with different constraints and incentives, will make different choices and this means that they will each have their own "set of states", with possibly only the spatial component in common. Through this subjective component, our approach of geographical distance makes a link to the domains of behavioral geography (Golledge 1997; Mark et al. 1999), and of the psychological and economic literature on travel behavior (Zahavi 1976; Mokhtarian and Salomon 2001; Acker, Wee, and Witlox 2010). In our framework, distance, as the solution of an optimization problem, is not universal: it actually depends on the constraints - linked to the context under which minimization occurs. In this sense we follow the views - inspired by Poincaré's reflections (1908, p. 116) - of modern human geographers (Blaut 1961; 
Gatrell 1983; Bailly 1985; Couclelis 1999). This approach moves away from an absolute - Newtonian and Kantian - conception of space and supports a more relative and subjective conception.

A related work is the Geographical Measurement Framework proposed by Miller and Wentz (2003) introducing geographic attributes that are attached to locations (stock) or to couples of locations (flows); these are spatial attributes. Our approach differs because our context is related to movement, to distances measurement, and not directly attached to space.

\section{Breaks and the triangle inequality}

In the next section, we shall introduce contextual metrics, ${ }^{1}$ which are metric-like functions defined on an enriched space modeling both physical space and a variety of possible factors influencing travel. To motivate and explain the need for this enrichment, let us discuss how the notion of break has been considered incorrectly as an impediment to the universal validity of the triangular inequality (L'Hostis 2016; L'Hostis 2017; L'Hostis 2020). The breaks in itineraries, that are necessary to relaunch movement, do not entail the idea of sub-optimality in distances that the triangle inequality violation suggest. In particular Huriot, Smith, and Thisse (1989, p. 313) admit that their proposed minimum cost distance may violate the triangle inequality. While they do not give an explicit example, it is plausible that what they have in mind is close to example 2.1 below.

One could develop a semi-metric structure that assumes a violation of the Triangle Inequality, possibly replaced by a less demanding inequality (Wilson 1931; Huriot, Smith, and Thisse 1989), even though this would entail losing major geometrical properties, the inability to use the spatial analytical techniques of Geography (Ahmed and Miller 2007), and entering abstract spaces (Wilson 1932, p. 517). However, our main motivation to develop a framework where we keep the Triangle Inequality is that it represent more accurately the very concept of distance as the least possible amount of effort needed to go from one location to another.

As extensively shown by L'Hostis (2016), several authors attach the idea of a human as an effort minimizer (Zipf 1949) to cost and "not just to distances" (Montello 1991, p. 113). We defend here an opposite view and propose to root the idea of optimization into the definition of geographical distance and its mathematical

\footnotetext{
${ }^{1}$ We shall use the word metric for the functions considered as a whole, and the word distance for individual measurements. So, if $d: X \times X \rightarrow[0,+\infty)$ is a metric and $p, q \in X$ are two locations, the number $d(p, q)$ shall be called the distance from $p$ to $q$.
} 


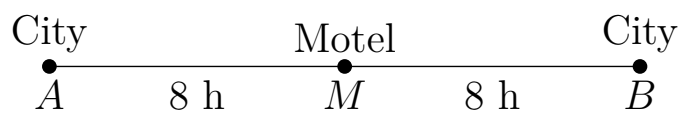

Figure 1: Two cities, a midway motel and the length of two paths $A M$ and $M B$

formalization. We refute the idea that geographical distance measurement could eventually be suboptimal. Departing from the point of view of Huriot, Smith, and Thisse (1989, p. 313), we claim that as soon as distances are well-defined through an optimization process, the triangle inequality is satisfied. Apparent violations of the triangular inequality are either a lack of optimization, as in the "two errors" debunked by L'Hostis (2016), or a lack of well-definiteness as we will explain below. The reinterpretation of the following simple example described by L'Hostis (2016) is at the core of our approach.

Example 2.1. The motel case Assume, as on figure 1, two cities $A, B$ are connected by a single road with a motel $M$ in between; to travel by car between $A$ and $M$, or between $M$ and $B$ takes 8 hours. Assume further that one is not able or allowed to drive for more than 8 hours in a row, and that after 8 hours of driving an 8 hours rest is needed. In a time-geography framework, the motel is a space-time anchor (Kuijpers, Miller, Neutens, et al. 2010) that compels the presence for an 8 hours period of time.

It has been claimed that this is a counter-example to the triangular inequality (L'Hostis 2016): the distance (in travel time) from $A$ to $B$ would be 24 hours (twice 8 hours of driving and 8 hours of rest in between), larger than the sum of the distance from $A$ to $M$ and the distance from $M$ to $B$ (both 8 hours). While L'Hostis has proposed as a solution to relax the triangular inequality, we argue that the problem here actually does not lie in the triangular inequality, but in the fact that distances (travel times) are not well-defined out of context: for example the distance from $A$ to $M$ can be either $16 \mathrm{~h}$ or $8 \mathrm{~h}$, depending on the necessity to consider the break time or not. Both measurement can make sense in a geographical context. For instance the time duration of long car trips provided by online routing services ${ }^{2}$ usually do not include rest time, which may be avoided if two drivers take turns along the journey. Going back to our example, imagine the motel manager has to go to $A$ fetch some commodities: neglecting the time involved in logistics, after her 8 hours drive to $A$ she cannot drive back straight away. She thus stands in $A$ with no way to be at $M$ until 16 hours later. The travel time between any two points in fact depends on whether the traveler is rested or tired: there is a context to be taken into account.

\footnotetext{
${ }^{2}$ For instance: https://maps.openrouteservice.org/
} 


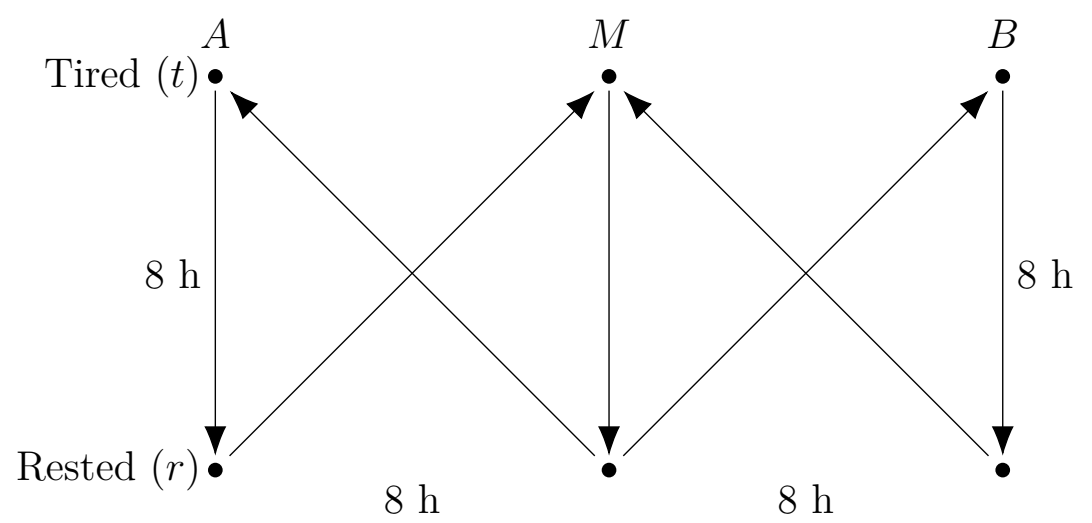

Figure 2: Two cities, a midway motel, and two contexts. Each arrow takes 8 hours.

More precisely, one can model the situation by a set of 6 couples, or states, where the context is written for cleaner notation in exponent of the location:

$$
\left\{A^{r}, A^{t}, M^{r}, M^{t}, B^{r}, B^{t}\right\} \simeq\{A, M, B\} \times\{r, t\}
$$

encoding the six possible combinations of a location and a context $(r$ for rested, $t$ for tired) of the traveler, as in figure 2. Each of the following actions take 8 hours: rest while staying in place, travel from a location to a neighboring one; traveling is only possible when rested and causes tiredness. Now the minimal length of a path between two states does satisfy the triangle inequality.

By enriching the space we see the initial model, where the triangle inequality seemed not to hold, as the mere shadow of a more accurate representation of the situation at hand. The apparent violation of the triangle inequality is revealed as an undeterminacy caused by important information being left out. Modeling much more general situations where non-spatial information, i.e. a context, influence the length of travel is the goal of the framework we are about to develop.

\section{Contextual metrics}

Definition 3.1. Let $X$ be a set, which shall represent the geographical space; elements of $X$ are called locations. Let $C$ be a set called the set of contexts. A pair in $X \times C$ corresponding to location $p$ and context $c$ will be called a state and denoted by $p^{c} ;{ }^{3}$ it compounds the information on the location $p$ and the context $c$.

\footnotetext{
${ }^{3}$ The usual mathematical notation would be $(\mathrm{p}, \mathrm{c})$, but a lighter notation seemed better and confusion with taking power is unlikely.
} 


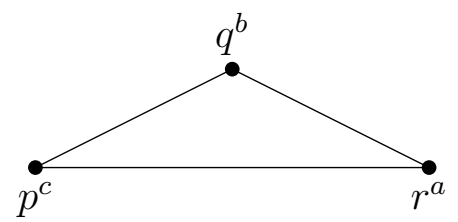

Figure 3: A triangle in the state space: each vertex is a state, i.e. a pair (location $^{\text {context }}$ ), and each edge is a path.

A contextual metric on $X$ (with context set $C$ ) is a function

$$
d:(X \times C)^{2} \rightarrow[0,+\infty]
$$

satisfying the following axioms:

i. (Identity) for all $p^{c} \in X \times C, d\left(p^{c}, p^{c}\right)=0$,

ii. (Separation In Space) for all $p, q \in X$, if $\inf _{c, b \in C} d\left(p^{c}, q^{b}\right)=0$ then $p=q$,

iii. (Triangle Inequality) for all $p^{c}, q^{b}, r^{a} \in X \times C$,

$$
d\left(p^{c}, q^{b}\right) \leq d\left(p^{c}, r^{a}\right)+d\left(r^{a}, q^{b}\right),
$$

The number $d\left(p^{c}, q^{b}\right)$ shall be called the contextual distance between the two states $p^{c}$ and $q^{b}$, so as to distinguish with the function $d$ as a whole; we may use the term "distance function" to denote functions that play a similar role to, but may not satisfy the properties of, a metric or a contextual metric. While a non-contextual distance is measured between two points in space, a contextual distance is measured between two states, i.e. between two pairs of a location and a context. A crucial point of differentiation between the contextual and the non-contextual distance resides in the fact the addition of two measurements must take into account the context: the Triangle Inequality is only assumed when the ending context of the first term is equal to the starting context of the second term.

The geographical interpretation is that the contextual distance $d\left(p^{c}, q^{b}\right)$ is meant to express the least possible disutility (e.g. least possible length traveled, time spent, monetary cost, etc.) needed to be endured when starting at location $p$ with context $c$, to attain location $q$ with context $b$. We will often speak of "length" instead of disutility, as we want to think about the enriched space geometrically, but it should be remembered that it can measured in various units.

A contextual metric is very similar to a metric on an "enriched space" $X \times C$, with the following differences: 
- we dropped symmetry altogether since contexts will often represent asymmetrical constraints, as in Example 2.1,

- separation is only in space, it could be costless to change the context in some ways,

- we allow the value $+\infty$ since some states could be inaccessible (e.g. when the context is an available budget and travel costs money, one cannot raise one's remaining budgets by traveling).

Note that given we have no symmetry, the order of the arguments matter: it might happen that $d\left(p^{c}, q^{b}\right)>d\left(p^{c}, r^{a}\right)+d\left(q^{b}, r^{a}\right)$. An example is given by the motel case 2.1: we have $d\left(A^{r}, B^{r}\right)=32 \mathrm{~h}$ but $d\left(A^{r}, M^{t}\right)+d\left(B^{r}, M^{t}\right)=16 \mathrm{~h}$.

From a contextual metric, one can construct several metric-like quantities on the geographical space $X$ itself, by taking extrema. They will be used in the subsequent examples to underline the properties of the contextual distance. The specific $\left(d^{c, b}\right)$, semi-specific $\left(d^{c, *}\right.$ and $\left.d^{*, b}\right)$, minimal and maximal distance functions defined by

$$
\begin{aligned}
d^{c, b}(p, q) & =d\left(p^{c}, q^{b}\right) & d^{c, *}(p, q) & =\inf _{b \in C} d\left(p^{c}, q^{b}\right) \\
d_{\min }(p, q) & =\inf _{c, b \in C} d\left(p^{c}, q^{b}\right) & d_{\max }^{*, b}(p, q) & =\sup _{c \in C} d_{c}(p, q)
\end{aligned}
$$

represent the least possible length of a traveling from location $p$ to location $q$, respectively when constraining both the starting context and ending contexts $\left(d^{c, b}\right)$, when constraining only one of them and disregarding the other $\left(d^{c, *}\right.$ and $\left.d^{*, b}\right)$, in the most favorable contexts at both ends $\left(d_{\min }\right)$, and finally in the worst case for the starting context, but without constraint on the arriving context $\left(d_{\max }\right)$.

Denoting by $p^{*}$ the set $\{p\} \times C$ of all states with location $p$, we thus have $d_{\min }(p, q)=d\left(p^{*}, q^{*}\right)$ in the usual mathematical sense of distance between sets. In the motel example 2.1, what had originally been considered as the distance between two locations is in our framework their minimal distance: it corresponds to a bestcase scenario and will in general not satisfy the Triangle Inequality. This is easily understood: that $d_{\min }(p, r)$ is small means that there exist some pair of states in $p^{*}$ and $r^{*}$ that are close to each other; and that $d_{\min }(r, q)$ is small means that there exist some pair of states in $r^{*}$ and $q^{*}$ that are close to each other; but if the states in $r^{*}$ do not match in the two pairs, it might be that all states of $p^{*}$ are far away from all states of $q^{*}$.

A simple verification shows that $d^{c, *}, d^{*, b}, d_{\min }$ and $d_{\max }$ satisfy the Identity and Separation property of usual metrics (e.g. $\forall p, q \in X: d_{\max }(p, q)=0$ if and only if 
$p=q$ ), while $d^{c, b}$ has the Separation property but might not satisfy the Identity property. Symmetry is not to be expected in general for either of $d^{c, b}, d^{c, *}, d^{*, b}, d_{\min }$ or $d_{\max }$, but can happen (e.g. in Example 2.1, $d_{\min }$ and $d_{\max }$ are symmetric even though $d$ is not). The same can be said about finiteness: in many cases we will see that $d^{c, b}, d^{c, *}, d_{\min }$ and $d_{\max }$ take only finite values, but this is not a general rule.

We have the following simple result showing that $d_{\max }$ is somewhat close to an actual metric, and we call it the maximal quasimetric.

Theorem 3.2. Let $d$ be a contextual metric on a space $X$ with set of contexts $C$. Its maximal quasimetric $d_{\max }$ satisfies the Triangle Inequality:

$$
\forall p, q, r \in X, \quad d_{\max }(p, q) \leq d_{\max }(p, r)+d_{\max }(r, q) .
$$

Note that given we have no symmetry, again the order of the arguments matter: it might happen that $d_{\max }(p, q)>d_{\max }(p, r)+d_{\max }(q, r)$.

Proof. Let $p, q, r$ be locations and consider any $\varepsilon>0$. By definition of the least upper bound, there exist $c \in C$ such that $d_{\max }(p, q) \leq d_{c}(p, q)+\varepsilon$. Then, there exist $a \in C$ such that $\left.d_{c}\left(p^{r}\right) \geq d\left(p^{c}\right), r^{a}\right)-\varepsilon$ and last $b \in C$ such that $d_{a}(r, q) \geq d\left(r^{a}, q^{b}\right)-\varepsilon$. Using these inequalities, the definitions of $d_{c}$ and $d_{\max }$ and the Triangle Inequality of $d$, we have:

$$
\begin{aligned}
d_{\max }(p, q) & \leq d_{c}(p, q)+\varepsilon \\
& \leq d\left(p^{c}, q^{b}\right)+\varepsilon \\
& \leq d\left(p^{c}, r^{a}\right)+d\left(r^{a}, q^{b}\right)+\varepsilon \\
& \leq d_{c}(p, r)+d_{a}(r, q)+3 \varepsilon \\
& \leq d_{\max }(p, r)+d_{\max }(r, q)+3 \varepsilon .
\end{aligned}
$$

Letting $\varepsilon$ go to zero, in the limit we get $d_{\max }(p, q) \leq d_{\max }(p, r)+d_{\max }(r, q)$.

Remark 3.3 (Contextual network). In many situations, for example the motel example discussed above 2.1 (Figure 2), it is convenient not to specify explicitly the whole contextual metric, but only the lengths of a set of "elementary movements", or arcs, forming a (contextual) network (the same remark applies to classical metrics). We shall give a detailed explanation of this formalism in Appendix A, but let us give here enough information to use it in simple cases below.

An arc will be defined by a starting state, and ending state, and a "length", or cost (measured in an arbitrary unit: it could be an actual length or a duration or a monetary cost or any other additive disutility); the notation $\alpha: p^{c} \rightarrow q^{b}$ shall mean 
that $\alpha$ is an arc starting at location $p$ in context $c$ and ending at location $q$ in context $b$. Its length shall be denoted by $\ell(\alpha)$. A finite sequence of arcs such that the ending state of each of them is equal to the starting state of the next shall be called a path, denoted as $\gamma=\alpha_{1} \alpha_{2} \ldots \alpha_{k}$, and we extend the length by additivity: $\ell(\gamma)=\sum_{i} \ell\left(\alpha_{i}\right)$. The starting state of $\gamma$ is the starting state $p^{c}$ of $\alpha_{1}$ and its ending state is the ending state $q^{b}$ of $\alpha_{k}$; we write $\gamma: p^{c} \leadsto q^{b}$. Given a set of arcs so defined, we define the associated contextual metric by

$$
d\left(p^{c}, q^{b}\right)=\inf _{\gamma: p^{c} \sim q^{b}} \ell(\gamma)
$$

Similarly, we can describe many classical metric spaces by a network of arcs, modeling elementary trips, with endpoints in $X$ instead of $X \times C$. In Appendix A, and prove that under natural conditions the function $d$ defined in this way is indeed a contextual metric (see Theorem A.11).

\section{The unifying power of contextual metrics}

While we introduced contextual metric with the motivation to better understand breaks in movement, it turns out they also provide a unified framework to model a variety of situations. First, of course, a distance on a space $d$ yields a contextual distance by taking $C$ a singleton, so that we only gain in generality. The following examples are meant to be crude and simple illustrations of this unifying power. We then hypothesize that contextual metrics are a suitable mathematical definition for the study of all geographical distances.

Example 4.1 (The simple motel case and variants). Let us revisit the motel example 2.1. We shall take $X=\{A, B, M\}$ and $C=\{r, t\}$ ( $r$ for "rested" and $t$ for "tired"). Travels are composed from two types of elementary arcs: $\alpha_{I J}: I^{r} \rightarrow J^{t}$ for the trip from location $I$ to the adjacent location $J$ and $\beta_{I}: I^{t} \rightarrow I^{r}$ for resting at location $I$; all arcs have length 8 hours. We then observe that neither $d_{\min }$ nor $d^{r, *}$ satisfy the Triangle Inequality; however the contextual metric $d$ itself satisfies the Triangle Inequality, and so does $d_{\max }$.

Assume more generally that we are given a metric space $(X, d)$ modeling driving times: the elements of $X$ are cities, and $d(I, J)$ is the minimal driving time from $I$ to $J$. Assume we want to take into account a legislation that imposes a resting time of duration $P>0$ after driving for a time $D>0$. This situation can be observed in the freight road transport with driving rules for lorry drivers (Chapelon 2006). D is the amount of fatigue in hours of driving that makes a break necessary or mandatory; 
in the case of freight road transport this level is fixed by professional rules, while in the domain of individual road transport this level are indicated by good practice. to take into account such constraints it suffices to introduce $C=[0, D]$, a context $c \in C$ representing a upper bound of the fatigue in hours of driving since the last rest; that is, being in the state $I^{c}$ means that we stand at $I$ and have not driven for more than $c$ hours since the last rest. Taking a upper bound allows for more flexibility: when one seeks to attain a location $J$ with fatigue $b$, attaining $J$ with lower fatigue is even better. Then the minimal time needed to get from $I^{c}$ to $J^{b}$, given the resting constraints, is

$$
\tilde{d}\left(I^{c}, J^{b}\right)=d(I, J)+k_{I J} P \quad \text { where } \quad k_{I J}=\left\lceil\frac{c+d(I, J)-b}{D}\right\rceil .
$$

(Here $k_{I J}$ represent the number of breaks needed.) The function $d$ is easily checked to be a contextual metric on $X \times C$. We remark that here, whenever $d$ is symmetric, $d_{\min }$ and $d_{\max }$ are both symmetric too.

A more complicated variant is obtained when rest is only possible at a location, not in between; or worse, resting is only possible at some locations. This situation is closer to the observed practices of lorry driving especially on expressways where stop is only allowed in dedicated service areas. A contextual metric can be constructed in a similar way as above, but the computation of contextual distances now needs the full contextual data: we cannot recover it from the initial distances only. Note that in some cases we can get non-symmetric contextual metrics. For example, service areas that are only accessible from one side of a motorway can break the symmetry.

The location of break points, or stations, is tightly related to the spatial patterns of trips and represents a highly relevant feature of geographical spaces. The caravanserais, playing the role of halting places, where the major infrastructure of the Silk Road (Williams 2014). The need for the first break for Parisian travelers to the south of France has fueled the economic specialization of Burgundy in the touristic sector around hotels and restaurants that shaped the reputation of the region (Bavoux 2009). The availability of - and the market served by - petrol stations on expressways is directly linked to the consumption of fuel by vehicles on the trunk lines. The location of charging stations is a key factor for the development of the use electric vehicles ( $\mathrm{Tu}$ et al. 2016). These examples show that a suitable framework caring for rest and energy reloading for movement sustaining, analyzed with the contextual distance, is of significant value for spatial analysis.

Example 4.2 (Multimodality and intermodality). Services such as Open Route 
Service $^{4}$ provide computation of minimal travel times, depending on the mean of transportation. This correspond simply to a family of metrics $\left(d_{T}\right)_{T \in \mathcal{T}}$ where $\mathcal{T}$ is the set of transportation means. We can include this in our framework by taking $\mathcal{T}$ as context set and defining the contextual metric by

$$
d\left(p^{T}, q^{S}\right)= \begin{cases}d_{T}(p, q) & \text { if } T=S \\ \infty & \text { otherwise }\end{cases}
$$

whenever $p, q \in X$ (the set of locations one can click on the map) and $T, S \in \mathcal{T}$. Then $d_{\text {min }}$ gives between any two places the time needed using the most efficient mean of transportation; it can violate the Triangle Inequality: if the most efficient means of transportation from $p$ to $r$ and from $r$ to $q$ are different, it can be that all means of transportation yield a travel time above $d_{\min }(p, r)+d_{\min }(r, q)$.

If we want to account for possible changes in mean of transportation, at least in some locations (e.g. take a rental bike at a station, enter the subway, etc.), it suffices to add arcs at these locations that correspond to such changes, and redefine $d$ allowing to combine travels through a given mean of transportation and change thereof. These new arcs should usually be given a non-zero length, corresponding to the time needed to operate the change.

Example 4.3 (Public transport timetables). This next example models travel using scheduled trips between various stations of a public transportation system, such as flights between airports, or trains or buses between stations. Our space $X$ will consist of finitely many stations, while our context set $C$ will be a subset of $\mathbb{R}$, considered as a time variable. Our network $N$ will contain two kinds of arcs: $N=T \cup W$ where

- each scheduled trip will correspond to an arc $f \in T$; we denote by $s_{X}(f)$ and $e_{X}(f)$ the departure and arrival stations of the flight service and by $s_{C}(f)$ and $e_{C}(f)$ the departure and arrival time of the trip (with of course $s_{C}(f)<e_{C}(f)$ ).

- for every station $x \in X$ and each couple $\left(t_{1}, t_{2}\right) \in \mathbb{R}^{2}$ such that $t_{1} \leq t_{2}$, there is an arc $w_{x, t_{1}, t_{2}}: x^{t_{1}} \rightarrow x^{t_{2}}$ which models waiting at station $x$ from time $t_{1}$ to $t_{2}$. We will denote by $W$ the set of arcs coming from waiting time.

Last, we shall consider a length function $\ell: T \cup W \rightarrow[0,+\infty)$ representing the quantity the traveler is willing to minimize, e.g. total time of travel. We extend it to paths additively and define the contextual metric $d$ by minimization, as explained in Remark 3.3 and in greater details in Appendix A.

\footnotetext{
${ }^{4}$ https://maps.openrouteservice.org/
} 
As in the previous case, asymmetry already arises from the network itself : our definition of $N$ ensures that every path $\gamma: x_{1}^{t_{1}} \leadsto x_{2}^{t_{2}}$ satisfies $t_{1}<t_{2}$. Hence for every two airports $x_{1}$ and $x_{2}$ and any two times $t_{1}$ and $t_{2}$ such that $t_{1}>t_{2}$, the contextual distance $d\left(x_{1}^{t_{1}}, x_{2}^{t_{2}}\right)$ will be infinite for every choice of length function $\ell$ whereas $d\left(x_{2}^{t_{2}}, x_{1}^{t_{1}}\right)$ can be finite. Hence except in very degenerate cases the contextual metric will not be symmetric. The semi-specific distance function $d^{t, *}$ need not be symmetric either, since the flights from $A$ to $B$ and $B$ to $A$ need not be synchronized. Even $d_{\min }$ and $d_{\max }$ need not be symmetric, since the waiting time at connections can be different for the return trip.

If we consider the length function $\ell$ corresponding to the duration of the trip, we immediately get $d\left(x_{1}^{t_{1}}, x_{2}^{t_{2}}\right)=t_{2}-t_{1}$ whenever there exist a path $x_{1}^{t_{1}} \leadsto x_{2}^{t_{2}}$, $d\left(x_{1}^{t_{1}}, x_{2}^{t_{2}}\right)=\infty$ otherwise: the context being tied to the disutility, there is some redundancy. In this example:

- the semi-specific distance $d^{t_{1}, *}\left(x_{1}, x_{2}\right)$ will be $T-t_{1}$ where $T$ is the first possible arrival time of a trip from $x_{1}$ to $x_{2}$ which leaves $x_{1}$ after time $t_{1}$ (if no such trip exists, $\left.d^{t_{1}, *}\left(x_{1}, x_{2}\right)=+\infty\right)$

- the semi-specific distance $d^{*, t_{2}}\left(x_{1}, x_{2}\right)$ will be $t_{2}-T$ where $T$ is the last possible departure time of a trip from $x_{1}$ to $x_{2}$ which attain $x_{2}$ before time $t_{2}$ (if no such trip exist, $\left.d^{*, t_{2}}\left(x_{1}, x_{2}\right)=+\infty\right)$,

- the minimal distance $d_{\min }\left(x_{1}, x_{2}\right)$ will be equal to the infimum of the duration of a trip from $x_{1}$ to $x_{2}$ (and will usually not satisfy the Triangle Inequality),

- the maximal quasimetric will have properties which depend a lot on the contextual network: in particular if for every flight $f \in F, s_{C}(f) \geq 0$ (no trip starts before time 0$)$, taking $C=\mathbb{R}$ would yield $d_{\max }\left(x_{1}, x_{2}\right)=+\infty$ whenever $x_{1}$ and $x_{2}$ are different. Here the modeling choices are important to obtain relevant distances.

Let us be more specific and consider 3 airports $x, y$ and $z$ represented in 4 such that:

- there is a flight from $x$ to $y$ taking off at 10 and landing at 11.

- there is a flight from $y$ to $z$ taking off at 12 and landing at 13 .

- there is a flight from $x$ to $z$ taking off at 12 and landing at 14 . 


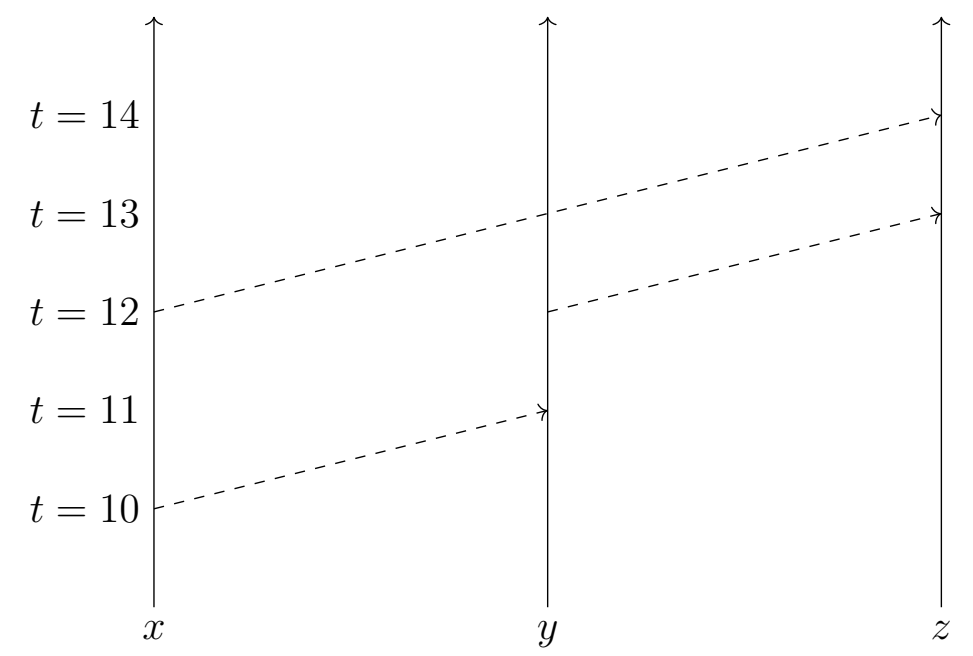

Figure 4: Representation of the contextual network from example 4.3. The space $X$ is represented horizontally while the context (here time) is represented vertically. Any subsegment of the vertical solid arrows is a valid arc in the network, while the dashed arcs cannot be subdivided.

Here the space $X$ is $\{x, y, z\}$, the context is $C=[9,15]$ considered as a time variable and the network $N$ is built from the timetable as described above. In this example:

$$
\begin{aligned}
& d_{\min }(x, z)=2, \quad d_{\max }(x, z)=+\infty \\
& d^{t, *}(x, z)=\left\{\begin{array}{ll}
13-t & \text { if } t \leq 10 \\
14-t & \text { if } 10<t \leq 12 \\
+\infty & \text { if } 12<t .
\end{array} \quad d^{*, t}(x, z)= \begin{cases}+\infty & \text { if } t \leq 13 \\
t-10 & \text { if } 13<t \leq 14 \\
t-12 & \text { if } 14<t .\end{cases} \right.
\end{aligned}
$$

The appearance of infinite distance should not be treated as a weakness of the example : it carries information. The infiniteness of $d^{t, *}(x, z)$ for $t>12$ shows that $z$ is not reachable if the trip starts later than time $t=12$.

Now if we assume the same flights take off every day, and change $C=\mathbb{R}$, we get:

$$
\begin{array}{ll}
d_{\min }(x, z) & =2 \\
d^{t, *}(x, z) & = \begin{cases}13-t & \text { if } t \in(-12,10] \\
14-t & \text { if } t \in(10,12]\end{cases} \\
d^{*, t}(x, z) & = \begin{cases}t-10 & \text { if } t \in[13,14) \\
t-12 & \text { if } t \in[14,37)\end{cases}
\end{array}
$$


and $d^{t, *}, d^{*, t}$ are periodic in $t$, of period 24 .

The computation of $d^{t_{1}, *}\left(x_{1}, x_{2}\right)$ and $d^{*, t_{2}}\left(x_{1}, x_{2}\right)$ for a real public transportation network, defined as time-dependant networks, is an important problem in practice and a very active field of research, see Müller-Hannemann et al. (2007) for timetable algorithms and Hall (1986), Delling et al. (2009), and Bast et al. (2016) for routing algorithms in time-dependant graphs. The semi-specific distances cover the main problems posed by time-dependant graphs. Note that two approaches can be found in this field: in the time extended approach the problem of computing $d^{t_{1}, *}\left(x_{1}, x_{2}\right)$ is tackled by choosing shortest path in the static graph whose vertices are states $x_{i}^{t_{i}}$, while in the time dependent approach the underlying graph has fixed vertices $x$ but dynamic edges which appear and disappear as time progresses according to available trips. In our framework they corresponds to considering either the contextual metric on the space $X \times \mathbb{R}$ of all states or the semi-specific distance function on the space $X$.

The length function we have presented here only takes into account the travel time, but the flexibility of our framework allows to take into account various criteria. One can for instance take as a length function the total price of the trip by assigning to each arcs in $W$ a length of 0 and each arc in $T$ the price of the ticket. One can also penalize connections by assigning to each possible trip $\alpha \in T$ a length $\ell(\alpha)=e_{C}(\alpha)-s_{C}(\alpha)+p$ where $p$ is a fixed penalty.

Example 4.4 (Minimizing duration under a constrained budget, or vice-versa). Another important situation is when one has a maximal available budget for one's travel, but still tries to minimize the total duration given this constraint. Then, $X$ being as usual the space to be considered, and $N$ be a non-contextual set of possible elementary trips (be it flights or train trips, etc.) with as before functions $s, e: N_{0} \rightarrow X$ indicating starting and ending locations, length function $\ell: N \rightarrow$ $[0,+\infty)$ representing the duration of each trip, and here an additional cost function $\$: N \rightarrow[0,+\infty)$. One can then set $C=[0,+\infty)$, a context $c \in C$ representing the remaining available budget, and

$$
\tilde{N}=\left\{\tilde{\alpha}_{c}: \alpha \in N, c \in C, c-\$(\alpha) \geq 0\right\},
$$

with the understanding that $\tilde{\alpha}_{c}: s(\alpha)^{c} \rightarrow e(\alpha)^{c-\$(\alpha)}$. We set $\tilde{\ell}\left(\tilde{\alpha}_{c}\right)=\ell(\alpha)$ and define from it the contextual metric $d$ as before. The quantity of interest is $d^{b, *}(p, q)$ where $b \in C$ is the given budget constraint, $p$ the starting location and $q$ the destination. A thorough analysis of such a model is conducted in Section 5, where it is shown that the monetary constraint can influence not only the distance (here, time of travel), but also the shortest paths and the shape of isochrones: context actually matters to how we move. 
Of course, one could want to minimize price under a time constraint. This is achieved by exchanging the roles of $\ell$ and $\$$, which makes no theoretical difference at all in our framework.

Remark 4.5 (Contextual distances for time geography). The above example directly implements key principles of time-geography; let us elaborate briefly on several linkages between our framework and time-geography.

Actually, the context-free framework of Section A.1 can be represented in the space-time cube (Kraak 2003; Anwar, Wei Zeng, and Arisona 2014) whenever the considered "length" is the duration of trips. The space-time is then given by the product $X \times[0,+\infty)$, with the second factor representing the time-length of paths.

Then, the addition of contexts makes it possible to introduce constraining or enabling quantities or properties in any number and of any nature. We thus obtain a "space-context-time" $X \times C \times[0,+\infty)$ with a non-spatial factor $C \times[0,+\infty)$ in which the last factor $[0,+\infty)$ has a prominent role: it encodes the quantity one seeks to minimize. Note that in the case where length is actually physical length of trips, it still makes sense to separate the factor $[0,+\infty)$ from the space $X$, as the latter will give the distances as the crow flies while the former will give the distance actually traveled, through the transportation network.

In example 4.4, the cost constraint is added, making $X \times C \times[0,+\infty)$ a "spacecost-time" cube. We may represent it in $3 \mathrm{D}$ by simplifying the space $X$ to a $1 D$ representation. The possibilities are endless, and in particular we insist that time may not always be the quantity to be minimized (so even in a simple context-free framework, one could use a "space-cost" cube).

\section{Context affects all aspects of movement: a simple toll expressway model}

We now introduce and analyse a theoretical geographical model with interesting features. It will show that a cost budget constraint can be encoded in our framework, and that taking context into account can influence movement significantly in the travelled length, in its possibility and in its shape.

Consider a straight toll expressway in an otherwise homogeneous and isotropic geographic space, assuming that movement on the expressway is twice as fast as outside it and that a toll proportional to the length drove on the expressway is due for using it. In this model we will consider paths that minimize the time spent, under the constraint of a monetary budget. This situation can be modeled in our framework as follows. 
The space is $X=\mathbb{R}^{2}$ and we assume the expressway follows the horizontal axis $\{(x, 0): x \in \mathbb{R}\}$. The set of contexts is $C=[0, \infty)$, a value $\beta \in C$ representing the available budget in monetary units for the toll (from now on, "budget" without specification shall refer to monetary budget). The network is decomposed as $N=$ $E \cup H$ where $E$ represents movement not using the expressway and $H$ movement along the expressway; we can set $E=\left\{\alpha_{p, q}^{E, \beta}: p, q \in X, \beta \in \mathbb{R}\right\}$ where $\alpha_{p q}^{E, \beta}$ represents the straight arc from $p$ to $q$ (we could include all differentiable arcs, but this would not change anything relevant) traveled with a starting monetary budget $\beta$ (which will also be the ending budget), and $H=\left\{\alpha_{x_{0}, x_{1}}^{H, \beta}: x_{0}, x_{1} \in \mathbb{R}, \beta \geq\left|x_{1}-x_{0}\right|\right\}$ where $\alpha_{x_{0}, x_{1}}^{H, \beta}$ represents the straight horizontal arc from $\left(x_{0}, 0\right)$ to $\left(x_{1}, 0\right)$ using the expressway, again with starting budget $\beta$ (here the ending budget will be lower due to the toll). Then assuming unit speed outside the expressway, we set (using $\|\cdot\|$ to denote the Euclidean norm):

$$
\begin{aligned}
& s\left(\alpha_{p, q}^{E, \beta}\right)=p^{\beta} \quad e\left(\alpha_{p, q}^{E, \beta}\right)=q^{\beta} \quad \ell\left(\alpha_{p, q}^{E, \beta}\right)=\|p-q\| \\
& s\left(\alpha_{x_{0}, x_{1}}^{H, \beta}\right)=\left(x_{0}, 0\right)^{\beta} \quad e\left(\alpha_{x_{0}, x_{1}}^{H, \beta}\right)=\left(x_{1}, 0\right)^{\beta-\left|x_{1}-x_{0}\right|} \quad \ell\left(\alpha_{x_{0}, x_{1}}^{H, \beta}\right)=\frac{1}{2}\left|x_{1}-x_{0}\right|
\end{aligned}
$$

where $\ell$ measures the time needed to travel the given arc. On the expressway the computed length in time is half that of a similar path in the rest of space. This example shows how our framework can be used to model a monetary budget constraint: use the context to encode the available budget, add as many arcs as needed to include the available budget and the various travel options, use the function $e$ to encode the cost of an arc, and in $N$ forbid any arc that cannot be paid for. This produces a subjective space, a space modelled from the point of view of an individual endowed of a given monetary budget.

Let us now analyze this model. For simplicity, we consider a fixed starting point $A=(0,1)$. The details of computations are not given, but they are all straightforward. First neglecting the budget constraint, to compute the distance between $A$ and a point $B=(x, y)$ one has to compare the straight segment $[A, B]$, of time cost $\|B-A\|=\sqrt{x^{2}+(y-1)^{2}}$, with the quickest path using the expressway. The path going from $A$ to $\left(x_{0}, 0\right)$, then taking the expressway to $\left(x_{1}, 0\right)$, then going straight to $B$ has total time cost

$$
\sqrt{x_{0}^{2}+1}+\frac{1}{2}\left|x_{1}-x_{0}\right|+\sqrt{\left(x-x_{1}\right)^{2}+y^{2}}
$$

Let us assume $x \geq 0$ (the other case following by symmetry), in which case it is clear that one should take $x_{0} \leq x_{1}$, both in the interval $[0, x]$. Then differentiating with respect to any of the $x_{i}$, one sees that the optimal choice is to enter and leave the 

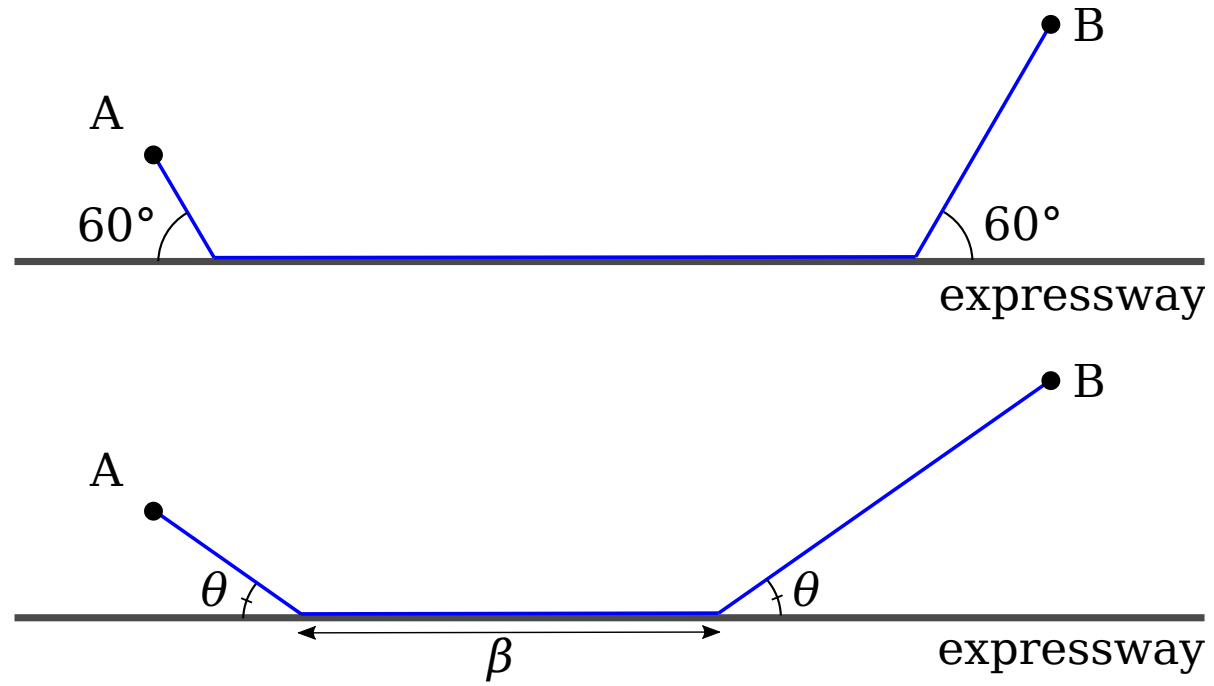

Figure 5: Optimal expressway use from $A$ to $B$ (top: unlimited budget, bottom: limited budget $\beta$ ).

expressway with a $60^{\circ}$ angle, see figure 5 . In other words, as soon as $x$ is large enough one should take $x_{0}=1 / \sqrt{3}$ and $x_{1}=x-|y| / \sqrt{3}$. Here "large enough" means larger than $(1+|y|) / \sqrt{3}$ in order to have $x_{1}>x_{0}$; for smaller $x$, taking the expressway is easily seen to be longer than going straight from $A$ to $B$. Then, we conclude:

Lemma 5.1. Whenever $|x|>(1+y) / \sqrt{3}$ and $\beta \geq|x|-(1+y) / \sqrt{3}$, the optimal path has time cost

$$
d^{\beta, *}(A, B)=d_{\min }(A, B)=\min \left(\sqrt{x^{2}+(y-1)^{2}}, \frac{3(1+|y|)}{2 \sqrt{3}}+\frac{x}{2}\right) .
$$

Moreover, if the minimum is realized by its first argument, then the straight segment $[A, B]$ is optimal; if the minimum is realized by its second argument, then the path using the expressway pictured in figure 5 (top) is optimal. If both arguments are equal, then both paths are optimal.

From this, it is easy to draw the isochrones from $A$; they are made of up to two circular arcs and four straight segments, see the left part of Figure 6; only the right side is shown given symmetry. This picture is similar to von Thunen's model of agricultural land use around a market city where a river takes the place of the expressway (Tobler 1993). This analysis is also consistent with the developments of the Von Thünen model with a road added to the homogeneous plain (O'Kelly 1989). 

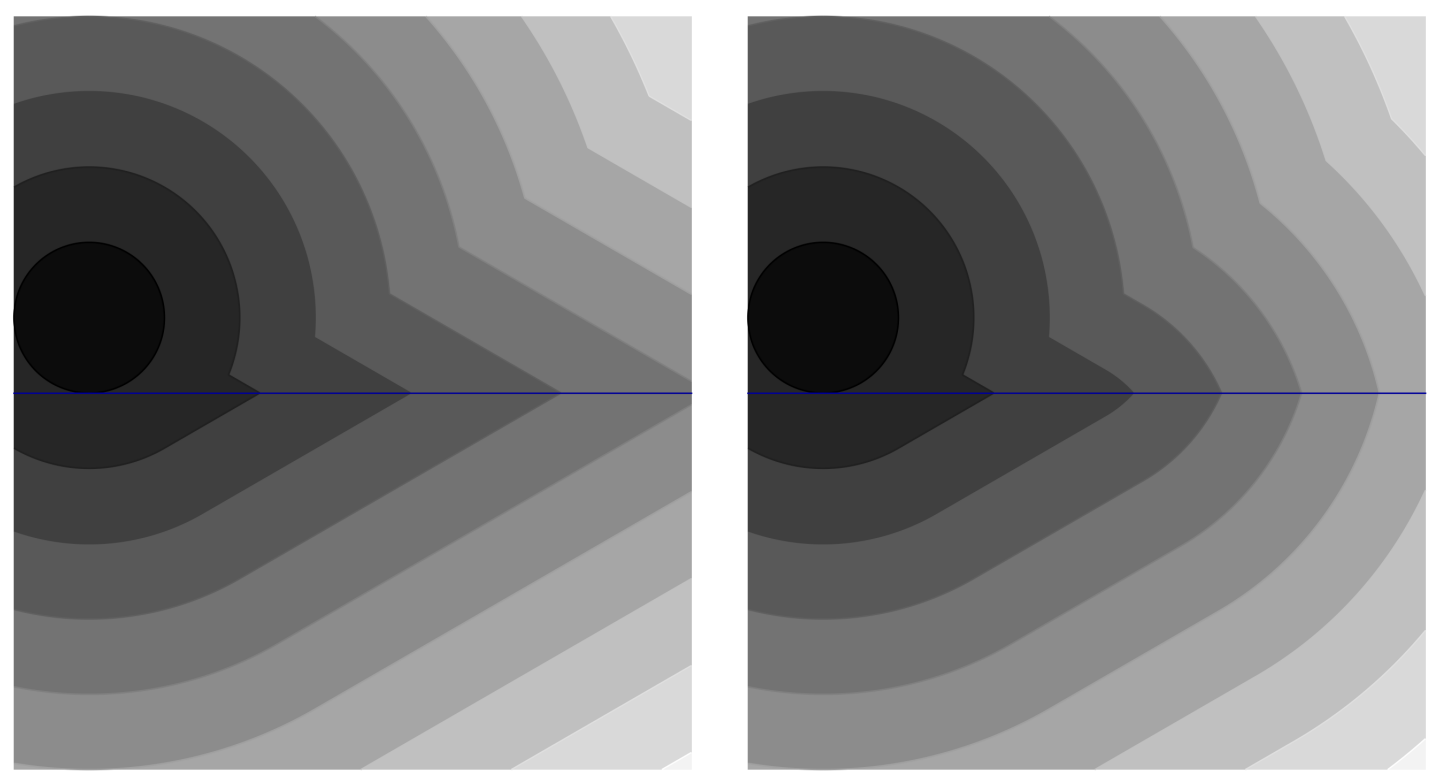

Figure 6: Isochrones from $A=(1,0)$ (left: large budget, right: limited budget $\beta=3$ ).

Now, let us take into account the budget limitation, in the regime where it matters i.e. $\beta<x-(1+y) / \sqrt{3}$ (again assuming by symmetry $x \geq 0$ ). Then the optimal expressway path is not available, as one is restricted to drive the expressway for a length at most $\beta$. If $B=(x, y)$ is such that even without budget constraint, the optimal paths is a straight Euclidean line, then the same must be true with a budget constraint. Assume now otherwise i.e. assume that driving the expressway was optimal without budget constraint; then using the expressway may or may not be optimal under the budget constraint. Let us thus first determine the optimal path among those using the expressway; the most time-effective way to proceed is then to spend all the budget, i.e. drive it for a length $\beta$. The last question is to determine where to enter and leave the expressway, and a differentiation argument shows that one should make the only choice making the entering and leaving angles equal. From there, one gets a new formula for the semi-specific contextual distance (no longer equal to the minimal contextual distance, since the budget constraint has kicked in).

Lemma 5.2. Whenever $|x|>(1+y) / \sqrt{3}$ and $\beta \leq|x|-(1+y) / \sqrt{3}$, the optimal path has time cost

$$
d_{\beta}(A, B)=\min \left(\sqrt{x^{2}+(y-1)^{2}}, \sqrt{(1+|y|)^{2}+(x-\beta)^{2}}+\frac{\beta}{2}\right) .
$$




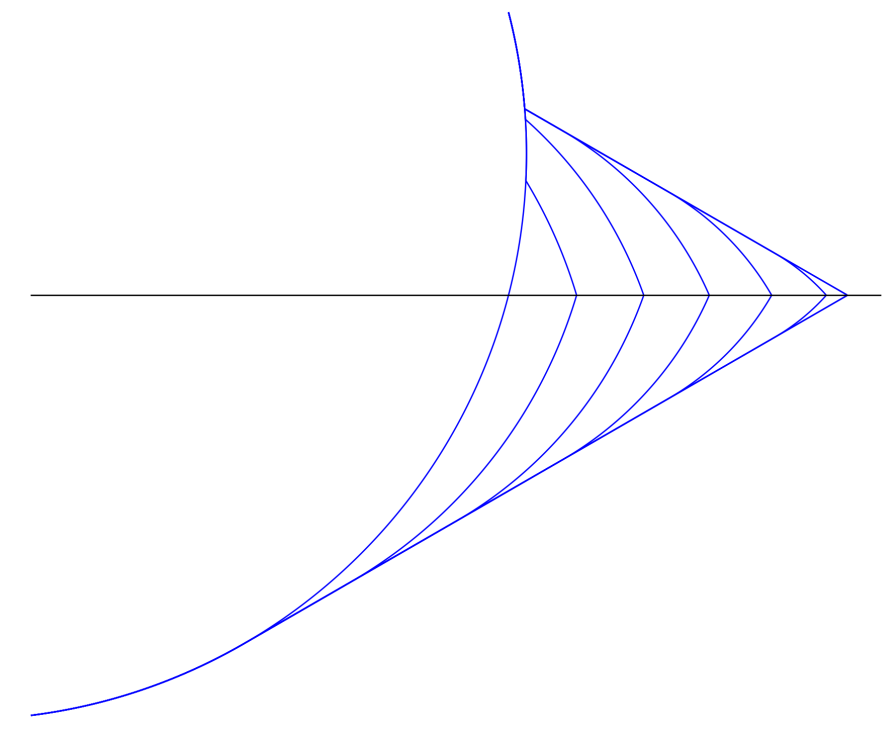

Figure 7: Isochrone from $A$ for time $t=4$, with varying budget $\beta$ from 0 (circle) to 8 (circle + triangle).

Moreover, if the minimum is realized by its first argument, then the straight segment $[A, B]$ is optimal; if the minimum is realized by its second argument, then the path using the expressway pictured in figure 5 (bottom) is optimal. If both arguments are equal, then both paths are optimal.

One can draw the isochrones given the budget constraint; for budget $\beta=3$ they are shown in the right picture of figure 6 . To see more clearly how budget influences isochrones, see figure 7 . We see that constraining budget makes the picture depart from von Thunen's modified by the river, and erodes the benefits of the expressway.

The drawing of isochrones above made apparent the obvious fact that a budget constraint change the region that can be attained in a given time. In particular, the time budget introduced by Zahavi (1976) gives access to a different variety of places depending on one's monetary budget allowing different transport options; more generally, a change of context (e.g. a more constrained monetary budget) may result in a change of the time needed to execute the given movement, and may therefore result in the movement not being executed (e.g. when the time required to move is too long). Context matters, and the analysis of accessibility could gain in precision and relevance by taking it into account. Let us point out, in this very simple example, other ways in which context is shown to influence all aspects of movement. 

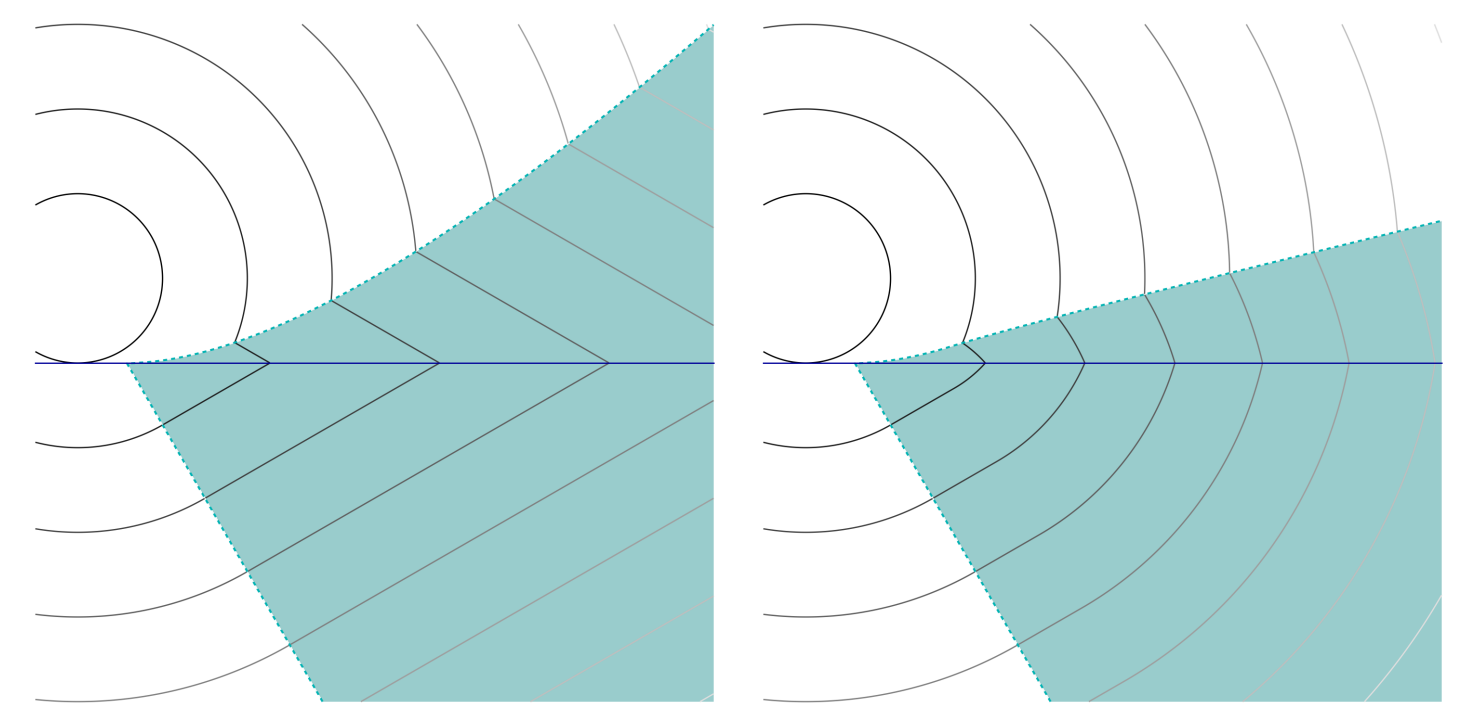

Figure 8: The region that is brought closer to $A=(0,1)$ by the expressway (left: unlimited budget; right: limiting budget $\beta=1$ ).

First, one can draw the region of all destinations for which the movement is influenced by the presence of the expressway (assuming the starting point is at $A$ ). This is pictured in figure 8 for unlimited budget (left) and limiting budget $\beta=1$ (right). We see that a budget constraint limits the impact of the expressway not only in travel time, but also in the destinations it helps reaching at all. Figure 9 shows how the influence of the expressway increases with available budget.

But there is more: a change of context can also result in a discontinuous change in how the movement is executed. Consider indeed the case of a traveler determined to go from the point $A=(0,1)$ to a fixed destination $B=(x, y)$ with $y>0$, assuming that $B$ lies in the region where taking the expressway can be beneficial (i.e. in the shaded area above the expressway in figure 8 , left). Now consider an available budget $\beta$ varying from $+\infty$ to 0 continuously; when $\beta$ is very large, the time-optimal path uses the expressway and the needed time for the travel does not at first depend on $\beta$. When $\beta$ goes under the threshold $\beta_{1}=|x|-(1+y) / \sqrt{3}$, the travel cannot use the expressway to full efficiency anymore and the time of travel starts increasing; but for now the optimal path varies continuously with $\beta$, with the angle $\theta$ at which the expressway is entered decreasing from $60^{\circ}$ (figure 5 ). When $\beta$ reaches the second threshold $\beta_{2}$ defined as the solution of the equation

$$
\sqrt{x^{2}+(y-1)^{2}}=\sqrt{(1+|y|)^{2}+(x-\beta)^{2}}+\frac{\beta}{2},
$$




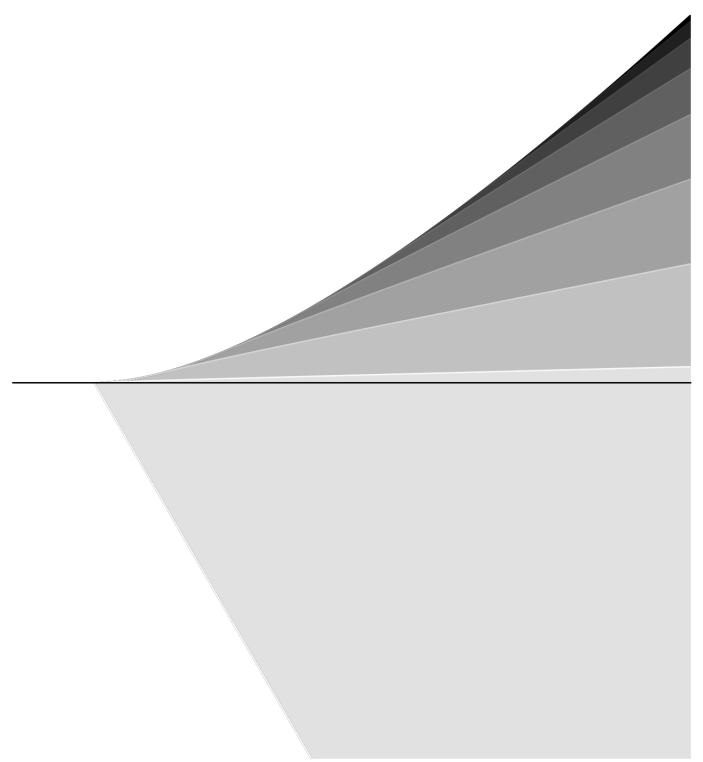

Figure 9: Comparison of the regions that are brought closer to $A=(0,1)$ by the expressway, when budget varies from .1 to 5 by increments of .7. Note that while the lower light grey region is brought closer to $A$ as soon as the budget is positive, the amount of time gained is sensitive to the budget, especially in its rightmost part.

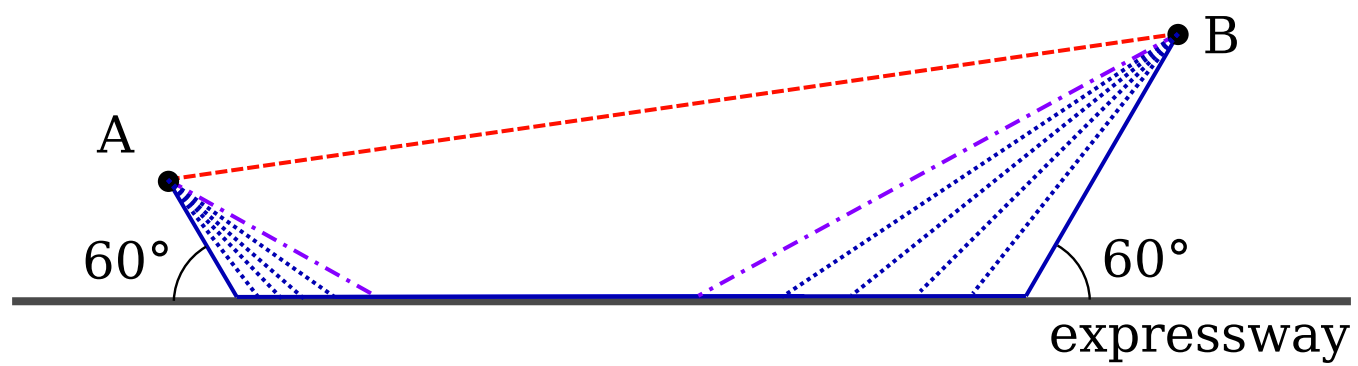

Figure 10: Comparison of the optimal paths from $A$ to $B$. Solid blue: $\beta \geq \beta_{1}$; dotted blue: $\beta \in\left(\beta_{2}, \beta_{1}\right)$; dashed red: $\beta<\beta_{2}$; dashed red and dash-dotted purple: $\beta=\beta_{2}$. 
there are two optimal paths: the first is by taking the expressway for a length $\beta=\beta_{2}$, and the other is a single straight line. When $\beta>\beta_{2}$, the single optimal path is close to the first one, but as soon as $\beta<\beta_{2}$, only the straight line is optimal (figure 10). There is therefore a discontinuity in the optimal path at $\beta=\beta_{2}$, even though the time needed to go from $A$ to $B$ varies continuously.
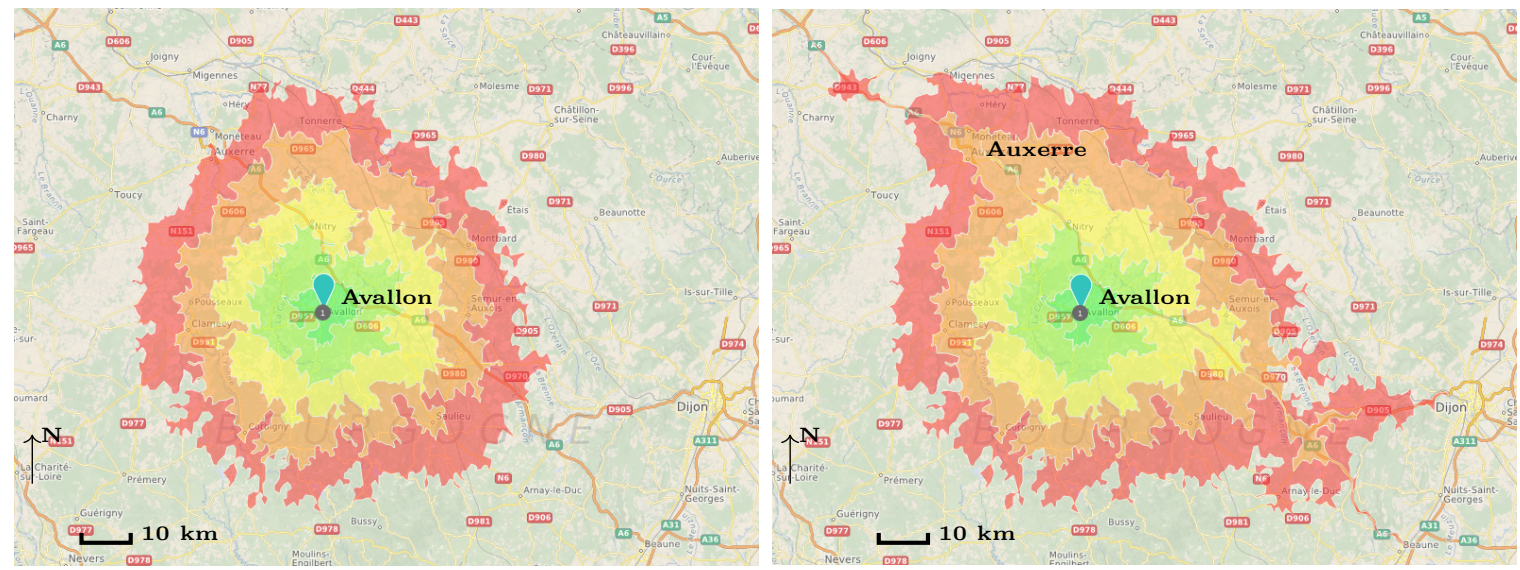

Figure 11: real life example - isochrones of 10 to 60 minutes driving, computed and rendered with OpenRouteService from Avallon (Yonne, France) with toll avoided (left) or accepted (right). In the toll case, in the north-west, the relatively high density of entrances to the expressway in the vicinity of the agglomeration of Auxerre make the fast infrastructure behave like a spatially continuous access road, as in our simplified example. By contrast, in the south-east, the discrete nature of the access to the expressway is visible through the discontinuous shape of the isochrone.

\section{Conclusion}

Our main contribution is the introduction of contextual metrics as a suitable framework to define geographical distances in a comprehensive way. While the values taken by a classical metric represent the least possible amount of displacement, or travel time, or more generally disutility, needed to go from one location to another, contextual metrics take moreover into account any contextual information relevant to travel, be they resources used for - or related to - movement or any other constraints. In our view, geographical distance is not exclusively a matter of geometry, it is also indissolubly related to - potential or actual - physical movement in geo- 
graphical space; every element entering the decision process guiding this movement needs to be taken into account in order to gain deeper understanding of it.

In appendix A, we shall provide a comprehensive framework that derives a distance or a contextual distance from a transportation network, with the novel property of accounting for both continuous and discrete networks on the same space. This shows more precisely how the Triangle Inequality is not an arbitrary assumption, but follows from first principles: a metric defined from optimized path necessarily respects the Triangle Inequality.

That it is a consequence of optimization explains why the Triangle Inequality lies at the heart of the concept of a metric. However breaks in movement, when they are made necessary i.e. by fatigue, an empty tank or legislation, do not contradict the principle of optimization, but induce apparent violation of the Triangle Inequality. Contextual metrics unravels this paradox, by framing the optimization problem in a space enriched by the context that the traveler has to take into account, making apparent that the violation of the Triangle Inequality was only an artefact of a model lacking crucial information. In the aim to reconcile the principle of optimization and the Triangle Inequality in the case of the break, we introduce and develop the idea of contextual distance, and we explore the different situations that can be modelled with this framework. The range of geographical situations tested underline the level of generalization that can be expected from this approach. Contextual distance seems hence suitable for describing all kinds of geographical distances.

Since contextual metrics respect the triangle inequality, they can be used within the context of spatial analysis in geography; they allow to consider altogether, in a geometrical framework, subjective aspects and objective aspects of space, movement and choices. We moreover proposed several quantities derived from contextual metrics (minimum, semi-specific and maximum distances between locations) that enrich the concept of geographical space.

As several of our examples show, contextual distances are a suitable way to introduce (among others) temporal constraints in the computation of geographical distances. The idea that time geography (Hägerstrand 1970) has exerted a strong influence on geography is quite consensual among scholars (Miller 2004a; Yuan 2009; Shaw and $\mathrm{Yu}$ 2009), even more if we consider the rarity of the expression of the opposing view (Merriman 2012). While time geography is focused on the interaction of space and time, its scope is broader: "In this way, the life paths become captured within a net of constraints, some of which are imposed by physiological and physical necessities and some imposed by private and common decisions. Constraints can become imposed by society and interact against the will of the individual." (Hägerstrand 1970) In this perspective, our approach can be seen as a progress toward the 
goal of bringing together the elements that drive movement. The flexibility of contextual distance makes them potentially useful in time geography, e.g. by considering monetary cost distances on the time-space to model the fact that moving at higher speed often comes at a higher cost, but also opens the possibility to extend concepts of time-geography beyond their current reach, e.g. by considering a cost-time-space accounting for an additional third quantity, see Remark 4.5.

To better show the relevance of this approach, in Section 5 we illustrate by a more substantial example how deeply the context affects various aspects of movement. The example is still pretty crude: we consider an expressway that allows for faster driving in exchange for a toll, in an otherwise homogeneous and isotropic flat landscape. Nonetheless, we show that the context (here, available budget) influences not only the time of travel from a point to another, but also the shape of isochrones and of shortest paths. We even show discontinuities in the shape of shortest paths: arbitrarily small changes in the available budget can make the shape of shortest paths "jump" from one shape to another, even though our model is continuous (continuous space, continuous time, and continuous toll). This demonstrates that changes in movement induced by the context alone, without modification in space or network, can be quite dramatic. With an additional time constraint added, the context may influence whether going from one point to another would be possible at all. We thus see that context has an effect on whether and how movement takes place. This model expresses a form of subjective space in the sense that the characteristics attached to the individual - formalized in the context - directly affect the optimum paths and hence geographical distances and the accessible geographical space.

Future works could examine the Triangle Inequality empirically. Time, kilometres and cost distances by various transport modes could be measured, and then tested regarding Triangle Inequality. The investigation could consider these datasets as contextual distances, adjusting contextual and non-contextual parameters, and test whether this mathematical framework allows to better understand the geometry of geographic spaces. In this direction, our affirmation that the Triangle Inequality is always satisfied opens more questions than it closes: first, in some spaces the Triangle Inequality is almost an equality for most triple of points while in other spaces a much stronger inequality is almost always true; this could be used as a geometric property classifying geographical spaces. Are there geographical spaces which seem very different from a geographic perspective but which share such geometric properties? On the contrary, are there geographical spaces that are thought of as close to each other, but in fact have very different geometric behavior in this respect?

Moreover, one could consider other inequalities, possibly involving more than three points. One could for example classify spaces by the constraints its geometry 
entails for the 6-tuple

$$
(d(p, q), d(p, r), d(p, s), d(q, r), d(q, s), d(r, s))
$$

when $(p, q, r, s)$ runs over all 4 -tuples of point in the given space. Searching various sets of geometric inequalities that, when verified by a given space, ensures the planar representation of this space up to some reasonable error would also be an interesting endeavour: it would inform map designer by telling them when no good map can exists, or rather how bad they must be, in a variety of contexts far beyond the mapping of Earth with its geometric distance. These findings could prove valuable in the domain of the representation of time-distances, where stress control is a key issue.

\section{A Path optimization and (contextual) metrics}

In this appendix, we explore in more detail the property of the metric-like functions obtained from minimizing the total cost (measured in a arbitrary unit) of paths in a network of arcs, first in the classical, context-free case, then in the contextual case. Compared with Huriot, Smith, and Thisse (1989), we shall give a more abstract representation of these paths in order for this model to better fit several common situations to be found in geographical spaces.

First, this model shall be usable to describe both continuous spaces and discrete spaces; e.g. road trips may be described, depending on scale or the chosen level of simplification, by continuous paths on the physical space, or by discrete paths on the graph defined by the road network.

Second this model shall be suitable to include, in a continuous space, plane flights or train trips, for which continuous paths are ill-suited: indeed passengers cannot step out of the plane or the flight during the trip, and even if the flight passes right above their very house, or the train track right next to it, they will have to end the trip and use another mean of transportation to go back home: "While in the air, [the flyer] is imprisoned in a narrow time-space tube without openings and he does not therefore effectively exist in the geographic locations over which he is flying." (Hägerstrand 1970) In a sense, even though physically planes and train do follow continuous curves, for all transportation matters the trips they permit are best described by discrete data: times and locations of departure and arrival. Additionally, this choice to accommodate for discrete and continuous trips in the same model will make it possible to work with only the locations that can be starting points or destinations of trips, which a mid-air point somewhere on a plane trajectory 
is not. We thus avoid the addition to the space of such irrelevant points, which would be implied in some situations by the use of only continuous trajectories.

\section{A.1 Networks and cost functions: the classical case}

Again, we are given a set $X$ called the space, whose elements are called locations; and in the modeling process one should only include in $X$ those places where people could go to or get from; in particular, $X$ might not represent the whole of the "physical space" that is being modeled.

A network on $X$ is a set $N$ together with two functions $s, e: N \rightarrow X$; elements of $N$ are called arcs, and given an arc $\alpha$ the locations $s(\alpha)$ and $e(\alpha)$ are called its starting point and endpoint respectively. In the modeling process, one should include in $N$ either all possible trips, or a set of elementary trips that will be enough to recover all of them by concatenation (see next paragraph). It is possible to define several arcs that correspond to the same displacement in the physical space, in order to account for other differences (e.g. traveling along a given fixed road by car, or on foot, or by bike could be modeled by defining three distinct arcs).

Both the space and the network can have finitely or infinitely many elements. For theoretical purposes it is important to allow even uncountable sets, in order to allow continuous spaces (see for instance Beguin and Thisse (1979, p. 333)). Of course, for any practical computation in continuous model one will make a discrete approximation, composed of finitely many locations and arcs.

Given a network $N$ on a space $X$, a path $\gamma$ from $p \in X$ to $q \in X$ is a finite word with letters in $N$ (i.e. an ordered tuple of arbitrary length, written $\gamma=\alpha_{1} \alpha_{2} \cdots \alpha_{k}$ for some $k \in \mathbb{N}$ and some $\left.\alpha_{i} \in N\right)$, such that $s\left(\alpha_{1}\right)=p, e\left(\alpha_{k}\right)=q$ and $e\left(\alpha_{i}\right)=s\left(\alpha_{i+1}\right)$ for all $i \in\{1,2, \ldots, k-1\}$. We also include as paths the trivial paths $\varnothing_{p}$ for each $p \in X$, which start and end at $p$ and are empty words (this necessitate to include the point $p$ in the data describing the path, which is unnecessary for non-empty paths). Given two paths $\gamma=\alpha_{1} \ldots \alpha_{k}$ and $\eta=\beta_{1} \ldots \beta_{\ell}$ such that $e\left(\alpha_{k}\right)=s\left(\beta_{1}\right)$, we define their concatenation as the path $\gamma * \eta:=\alpha_{1} \ldots \alpha_{k} \beta_{1} \ldots \beta_{\ell}$. A path is thus a concatenation of arcs, and represents a trip possibly made of a combination of elementary trips. The set of paths shall be denoted by $N^{*}$, and we shall write $\gamma: p \leadsto q$ to express that $\gamma$ is a path from $p$ to $q$. Note that despite the notation, $N^{*}$ depends on $s, e$ as much as it depends on $N$.

A length function on a network $N$ is simply a function $\ell: N \rightarrow(0,+\infty)$ (we could have included the value 0 , but this would have made the introduction of an additional adjective necessary later on). We immediately extend the function $\ell$ to a 
function defined on $N^{*}$, still denoted by $\ell$, by

$$
\ell\left(\alpha_{1} \ldots \alpha_{k}\right)=\sum_{i=1}^{k} \ell\left(\alpha_{i}\right)
$$

with the usual convention that an empty sum is zero, i.e. $\ell\left(\varnothing_{p}\right)=0$ for all $p \in X$. (Another equivalent way to formalize the same framework would be to consider a function $\ell: N^{*} \rightarrow[0,+\infty)$ asked to be additive, i.e. $\ell(\gamma * \eta)=\ell(\gamma)+\ell(\eta)$ for all paths $\gamma, \eta)$. The quasimetric on $X$ defined by $(N, \ell)$ is then set as

$$
d(p, q)=\inf _{\gamma: p \sim q} \ell(\gamma) .
$$

Note that this is not necessarily a metric unless we make additional assumptions; it can even take the value $+\infty$, whenever there are no paths from $p$ to $q$.

The term length function should be taken with a grain of salt: $\ell$ could be about any effort or disutility function (length, travel time, monetary cost, etc.) In some cases, it could be argued that disutility should be allowed to be sub-additive; the reader can check that most of the properties below would not be altered by this relaxation. In any case, sub-additive disutility can always be modeled in the present framework by adding an arc for each trip combination that allows for some savings.

\section{A.2 Properties of quasimetrics defined by a network}

We will now explore assumptions that ensure the various axioms defining a metric. The most troublesome is distinguishability, more precisely that different points should be at positive distance from each other. The following example shows what could go wrong.

Example A.1. Take $X=\{p, q\}, N=\left\{\alpha_{i}: i \geq 1\right\}$ with $s\left(\alpha_{i}\right)=p, e\left(\alpha_{i}\right)=q$, and $\ell\left(\alpha_{i}\right)=1 / i$ (we could enlarge $N$ to get symmetry and finiteness of the distance but this would mostly obscure the point). Then we have an infinite sequence of paths (each consisting of only one arc, $\alpha_{i}$ ) from $p$ to $q$, of length $1 / i$. Since this goes to zero as $i$ goes to infinity, we get $d(p, q)=0$.

Example A.2. Let us give a second example that feels less ad hoc. Take $X=[0,1]$, $N=\left\{\alpha_{p}^{q}:(p, q) \in[0,1] \times[0,1]\right\}$ with $s\left(\alpha_{p}^{q}\right)=p, e\left(\alpha_{p}^{q}\right)=q$ (we have one arc for each possible pair of starting and ending points) and $\ell\left(\alpha_{p}^{q}\right)=|p-q|^{2}$. Then for each $n \in \mathbb{N}$, the path

$$
\gamma_{n}:=\alpha_{0}^{1 / n} \alpha_{1 / n}^{2 / n} \cdots \alpha_{(n-1) / n}^{1}
$$

goes from 0 to 1 and has length $\ell\left(\gamma_{n}\right)=n \times(1 / n)^{2}=1 / n$. It follows $d(0,1)=0$. 
(Note that in both examples, the distance can only be approximated by the length of path, there are no given path linking the given points whose length is their distance. This phenomenon can occur even in cases where all axioms of a metric hold true.)

This leads us to the following definition:

Definition A.3. We say that the length function is non-degenerate when there exist a function $m:\{(p, q): p, q \in X, p \neq q\} \rightarrow(0,+\infty)$ such that for all $p \neq q \in X$ and all $\gamma: p \sim q$ we have $\ell(\gamma)>m(p, q)$.

This is somewhat trivial as it is tuned to exactly ensure distinguishability, but is still an operational definition as it leads one to find a lower bounding function $m$, which in most cases of interest shall be easy to either find or at least prove into existence. There are two particularly simple cases ensuring non-degeneracy.

Proposition A.4. Let $X$ be a space and $N$ be a network on $X$.

i. If for all $p, q \in X$ the set $\{\gamma: p \leadsto q\}$ of paths from $p$ to $q$ is finite, then every length function is non-degenerate.

ii. Let $\ell$ be a length function such that for some $\varepsilon>0$, for all arc $\alpha$ it holds $\ell(\alpha) \geq \varepsilon$. Then $\ell$ is non-degenerate.

Proof. Note that a length function $\ell$ is assumed to be positive on each arc, and is thus positive on each non-trivial path. When $\{\gamma: p \rightarrow q\}$ is finite, one can take $m(p, q)=\min _{\gamma: p \sim q} \ell(\gamma)$ which is positive as soon as $p \neq q$.

The second case actually assumes $\inf _{N} \ell \geq \varepsilon>0$, which implies that each nontrivial path has length at least $\varepsilon$ : one can then take $m(p, q) \equiv \varepsilon$.

Theorem A.5. Let $X$ be a space endowed with a network $N$ and a length function $\ell$, and denote by $d$ the corresponding metric, as defined by (2).

i. (Finiteness) For all $p, q \in X$, we have $d(p, q)<+\infty$ if and only if there exists $\gamma: p \leadsto q$.

ii. (Symmetry) If for all arc $\alpha$ there exist an arc $\alpha^{\prime}$ such that $s\left(\alpha^{\prime}\right)=e(\alpha)$, $e\left(\alpha^{\prime}\right)=s(\alpha)$ and $\ell\left(\alpha^{\prime}\right)=\ell(\alpha)$, then $d(p, q)=d(q, p)$ for all $p, q \in X$.

iii. (Triangle inequality) $d(p, r) \leq d(p, q)+d(q, r)$ for all $p, q, r \in X$.

$i v$. (Distinguishability) The property $(d(p, q)=0$ if and only if $p=q)$ holds if and only if $\ell$ is non-degenerate. 
In particular, assuming that every pair of locations is linked by a path, that each arc has a reverse arc of the same length (case of a symmetric network), and that the length function is non-degenerate ensures that $d$ is a metric. While among the three axioms defining a metric, the Triangle inequality is probably the one most discussed, we see as in Huriot, Smith, and Thisse (1989) that it is the one that needs the less hypotheses: it follows from the optimization of paths used in the defining equation (2).

Proof. The first point follows from the fact that $\ell$ does not take the value $+\infty$, so that $d(p, q)=+\infty$ if and only if the infimum defining it is over the empty set.

To prove the second point we consider $p, q \in X$ and prove $d(q, p) \leq d(p, q)$; equality follows by exchanging the roles of $p$ and $q$. If $d(p, q)=+\infty$, this is obvious. Otherwise, given any $\varepsilon>0$ there exist a path $\gamma=\alpha_{1} \alpha_{2} \cdots \alpha_{k}$ from $p$ to $q$ with $\ell(\gamma) \leq d(p, q)+\varepsilon$. Then, using the notation $\alpha_{i}^{\prime}$ for the reverse arc of $\alpha_{i}$ provided by the hypothesis, $\gamma^{\prime}:=\alpha_{k}^{\prime} \alpha_{k-1}^{\prime} \cdots \alpha_{1}^{\prime}$ is a path from $q$ to $p$ and

$$
\ell\left(\gamma^{\prime}\right)=\sum_{i=1}^{k} \ell\left(\alpha_{k-i}^{\prime}\right)=\sum_{i=1}^{k} \ell\left(\alpha_{k_{i}}\right)=\ell(\gamma) \leq d(p, q)+\varepsilon .
$$

Letting $\varepsilon$ go to zero, it follows $d(q, p) \leq d(p, q)$.

To prove the third point, let $p, q, r \in X$. If there is no path from $p$ to $q$ or no path from $q$ to $r$, then the right-hand side is $+\infty$ and the inequality is trivially true. Assume otherwise and let $\varepsilon>0$ be arbitrary. There exists paths $\gamma_{1}: p \leadsto q$ and $\gamma_{2}: q \leadsto r$ such that $\ell\left(\gamma_{1}\right) \leq d(p, q)+\varepsilon$ and $\ell\left(\gamma_{2}\right) \leq d(q, r)+\varepsilon$. Then $\gamma_{1} * \gamma_{2}: p \leadsto r$ so that

$$
d(p, r) \leq \ell\left(\gamma_{1} * \gamma_{2}\right)=\ell\left(\gamma_{1}\right)+\ell\left(\gamma_{2}\right) \leq d(p, q)+d(q, r)+2 \varepsilon .
$$

Letting $\varepsilon$ go to zero, we get the triangular inequality.

Concerning the fourth and last point, note that we always have $d(p, p)=0$ since the empty path $\varnothing_{p}$ has by definition length 0 . Assume now that $\ell$ is non-degenerate and let $m$ a function as given in Definition A.3; then whenever $p \neq q \in X$, for all $\gamma: p \leadsto q$ we have $\ell(\gamma) \geq m(p, q)$, so that $d(p, q) \geq m(p, q)>0$. Conversely, if $p \neq q \Longrightarrow d(p, q)>0$, then letting $m(p, q)=d(p, q)$ yields non-degeneracy of $\ell$.

\section{A.3 Examples}

Let us give a few examples showing how the above framework can be used to model various geographically relevant situations. 


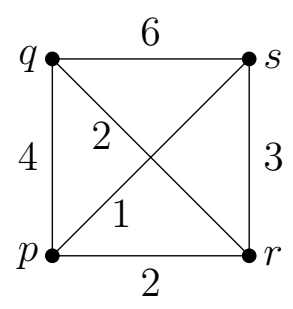

Figure 12: Four cities linked by six roads with time measurements from Haggett (2001, p. 341)

Example A.6 (Haggett's four cities, the usual graph case). Let us start with a discrete example. We choose the widely known example of Haggett (2001, p. 341): $X=\{p, q, r, s\}$ is a set of four cities, linked two by two by six roads that can be traveled in times given in figure 12 . These connections can be modeled in our framework by a network of twelve $\operatorname{arcs} N=\left\{\alpha_{i j}: i, j \in X\right\}$ (including both directions for each road) with $s\left(\alpha_{i j}\right)=i$ and $e\left(\alpha_{i j}\right)=j$; and by the length function (expressed in the unit of one hour)

$$
\begin{array}{lll}
\ell\left(\alpha_{p q}\right)=\ell\left(\alpha_{q p}\right)=4 & \ell\left(\alpha_{p r}\right)=\ell\left(\alpha_{r p}\right)=2 & \ell\left(\alpha_{p s}\right)=\ell\left(\alpha_{s p}\right)=1 \\
\ell\left(\alpha_{q r}\right)=\ell\left(\alpha_{r q}\right)=2 & \ell\left(\alpha_{q s}\right)=\ell\left(\alpha_{s q}\right)=6 & \ell\left(\alpha_{r s}\right)=\ell\left(\alpha_{s r}\right)=3 .
\end{array}
$$

Up to now, the model fits exactly Haggett's example. However, considering paths we see that what we call the distance becomes quite different; in particular, the road $\alpha_{q s}$ becomes irrelevant as the paths $\gamma_{1}:=\alpha_{q r} \alpha_{r s}$ and $\gamma_{2}:=\alpha_{q p} \alpha_{p s}$ each have cost 5 , less than the cost 6 of the direct arc $\alpha_{q s}$. This translates the fact that a traveler can go from $q$ to $s$ in only five hours, by avoiding the direct road. As underlined by L'Hostis (2016), optimization ensures the validity of the triangular inequality.

More generally, any graph with positive labels on its edges can be translated into the present framework, and the distance is the usual path-minimizing distance (actually, this case is a very classical framework in graph theory).

Example A.7 (Paths on continuous surface). Let us now show how the above framework can be used to model a continuous space. Let $X$ be a domain of the plane $\mathbb{R}^{2}$ (endowed with its canonical scalar product), meant to represent a region that is small enough to neglect the curvature of Earth. Assume that we are to model e.g. bird or pedestrian movement and that the region is homogeneous, without obstacles or roads facilitating certain trips compared to other.

Then it makes sense to consider as arcs all continuously differentiable curves $\alpha:\left[T_{0}, T_{1}\right] \rightarrow X\left(\right.$ where $\left.T_{0}<T_{1} \in \mathbb{R}\right)$, with $s(\alpha)=\alpha\left(T_{0}\right)$ and $e(\alpha)=\alpha\left(T_{1}\right)$, and to 
take the classical length function

$$
\ell(\alpha):=\int_{T_{0}}^{T_{1}}\left\|\alpha^{\prime}(t)\right\| \mathrm{d} t
$$

Then paths can be identified with continuous, piecewise continuously differentiable curves in an obvious way. Note that if we preferred to have "length" be physically homogeneous to a time, we could have written

$$
\ell(\alpha):=\int_{T_{0}}^{T_{1}} R\left\|\alpha^{\prime}(t)\right\| \mathrm{d} t
$$

where $R$ is the inverse of the speed of the modeled traveler. This number can be factored into a global scale for our model, and we disregard this consideration for now.

If $X$ is convex, then the quasimetric obtained by optimizing the length of curves coincides with the Euclidean metric: $d(p, q)=\|p-q\|$, and the unique shortest path from $p$ to $q$ is the line segment between these points (parameterized in any one-to-one way). Note that convexity of $X$ is necessary for this statement to hold: for example in an annulus formed by a large disk with a smaller disk removed, pairs of points separated by the "hole" cannot be joined by a line segment and their distance is greater than the Euclidean one.

Even without convexity, it is well-known and easy to check that $d$ is a metric if and only if $X$ is connected by continuous, piecewise continuously differentiable paths (otherwise, only the finiteness fails). In particular, non-degeneracy is easily proven by taking $m(p, q)=\|q-p\|$.

Example A.8 (Path on a non-homogeneous surface). More general situations can be modeled in a similar way than in Example A.7, for example by introducing inhomogeneity in the formula of the cost of an arc

$$
\ell(\alpha)=\int_{T_{0}}^{T_{1}} R\left(\alpha_{t}\right)\left\|\alpha^{\prime}(t)\right\| \mathrm{d} t
$$

where $R: X \rightarrow(0,+\infty)$ represent the inverse of the maximal speed at which one can travel at the point, which can be constrained e.g. by the roughness of the terrain or, as in (Angel and Geoffrey Hyman 1976), by traffic congestion (in mathematics, this is called a conformal change of Riemannian metric). We shall denote by $d_{R}$ the induced quasimetric (which is a metric in many cases, e.g. whenever there exist some $\varepsilon>0$ such that $R(p) \geq \varepsilon$ ). Such a situation is easily pictured using shades of gray to represent $R$, travel being more difficult in darker regions than in lighter ones. In 
the domain of geographical cartography, a similar representation of distances takes the form of cost-of-passage surface or cost surface (Angel and Geoffrey Hyman 1976; Beguin and Thisse 1979; Collischonn and Pilar 2000; Miller and Wentz 2003; White and Barber 2012). Related representations include Bunge proposing a crumpled space model to describe a marsh area difficult to cross surrounding a road (1962, p. 271). Representations with the same aim of describing different geographical time-distances use graphs in two (Plassard and Routhier 1987; Tobler 1997) or three dimensions (Mathis 1990; Mathis, Polombo, and L'Hostis 1993; L'Hostis 2009). One could also model general surfaces with Riemannian metrics (i.e. metrics which, at a very local scale, are Euclidean up to order 1 approximations); but note that the marvelous uniformization theorem from the early $\mathrm{xx}^{\text {th }}$ century shows that any such surface which is homeomorphic to a domain in the plane, can be represented by a distance $d_{R}$ obtained as above isometrically (i.e. in a way that distances are perfectly preserved). In particular, any part $Y$ of the earth that is not the whole can be represented in the plane by a map $\varphi: Y \rightarrow X \subset \mathbb{R}^{2}$, and a function $R$ can be chosen, in a way that $d_{R}(\varphi(p), \varphi(q))$ is exactly equal to the shortest path distance in $Y$ between $p$ and $q$. For a detailed historical and mathematical account of the uniformization theorem, the reader can consult de Saint-Gervais (2016).

Example A.9 (Mixing continuous and discrete spaces). The above examples are all classical in the geometry of metric spaces, and the only advantage of our framework seems to unify the discrete and continuous models. Let us now give an example, relevant in geography, that mixes both continuous and discrete aspects, and that we claim is quite satisfactorily modeled in our framework.

Let again $X$ be a domain in $\mathbb{R}^{2}$, convex say, and assume we have two ways to travel in $X$ : by car, where the constraints of the road network are assumed to be negligible, so that we modeled car travel as in Example A.7; and by plane, with exactly two airports located at $a_{1}, a_{2} \in X$, with a fast two-ways connection between them.

The network $N$ shall now be the union of the set $N_{g}$ of all continuously differentiable arcs on $X$ in the one hand, and of the set of the two connexions between the airports $N_{f}=\left\{\alpha_{f: 12}, \alpha_{f: 21}\right\}$ ( $g$ stands for "ground" and $f$ for "flight"). The starting and endpoints of arcs in $N_{g}$ are defined as usual, while the endpoints of the two arcs of $N_{f}$ are defined by $s\left(\alpha_{f: 12}\right)=e\left(\alpha_{f: 21}\right)=a_{1}$ and $s\left(\alpha_{f: 21}\right)=e\left(\alpha_{f: 12}\right)=a_{2}$.

The length function is given for $\alpha \in N_{g}$ by the physically homogeneous formula of Example A.7

$$
\ell(\alpha):=\int_{T_{0}}^{T_{1}} R\left\|\alpha^{\prime}(t)\right\| \mathrm{d} t
$$

where $R$ is the inverse of the speed of ground travel; and assuming both aerial 
connexions take the same time $T_{f}, \ell\left(\alpha_{f: 12}\right)=\ell\left(\alpha_{f: 21}\right)=T_{f}$.

Now, applying the framework above defines paths that can combine car trips with flights, and enable to construct the underlying optimized quasimetric. Here, provided $R>0$ and $T_{f}>0$, it is a metric and shortest paths are either line segments, or combination of one or two line segments and one flight.

Of course, more complicated situations with more airports, more connexions (not necessarily between all possible pairs of airports), possibly varying ground travel difficulty, can be modeled in the same way. One can also model alternative ground transportation means by taking $N_{g}=N_{g b} \cup N_{g c}$ where $N_{g b}$ and $N_{g c}$ are two disjoint copies of the set of continuously differentiable curves, the first ones corresponding to bike trips and the second ones corresponding to car trips. Then one sets

$$
\begin{array}{ll}
\ell(\alpha):=\int_{T_{0}}^{T_{1}} R_{b}\left\|\alpha^{\prime}(t)\right\| \mathrm{d} t \quad \forall \alpha \in N_{g b} \\
\ell(\alpha):=\int_{T_{0}}^{T_{1}} R_{c}\left\|\alpha^{\prime}(t)\right\| \mathrm{d} t \quad \forall \alpha \in N_{g c}
\end{array}
$$

where $R_{b}$ is the inverse of the biking speed, and $R_{c}$ is the inverse of the driving speed. As such, if we make the reasonable assumption $R_{b}>R_{c}$, the introduction of biking does not change the distance as it is a slower mean of transport than car (this is a case where introducing context would be useful to model possible unavailability of a car for some agents). But if one turns $R_{b}$ and $R_{c}$ into functions, with $R_{b}(p)<R_{c}(p)$ when $p$ lies in some regions, or if one changes the formula of the cost function to take into account the economical and environmental cost of $\mathrm{CO}_{2}$ emissions, then this enriched model becomes relevant.

Note that compared to Smith (1989), the present approach does notably not include axiom N3 (subpath closure) of Smith's definition 3.1: arcs need not be restrictable into subarcs, and this better represent flights or any other trips that cannot in practice be decomposed, such as train trips - one cannot jump off train between stations. On the other hand, Smith's path networks are particular cases of our definition of networks.

\section{A.4 Contextual networks and distances}

We shall now combine networks and contexts. We start as in Section 3 with a set of states $X \times C$ where $X$ is a set called the space, whose elements are locations, and $C$ is the context set; an element $p^{c}$ of $X \times C$ is called a state, it compounds the data of the location and the context. As before, $X$ should contain only those points of the physical space that are meaningful locations; $C$ should be made rich enough to 
model all elements of context that are relevant to the movement (affecting e.g. its length or its mere possibility).

\section{A.4.1 Contextual networks}

Contextual networks will be defined almost exactly as networks, but on the set of states. We still repeat the definitions as the different roles played by $X$ and $C$ will have an importance in the modeling process and in the interpretation. The case of a singleton $C=\left\{c_{0}\right\}$ will correspond to the above framework, while in example 2.1 we could take $C=\{r, t\}$ ( $r$ for "rested", $t$ for "tired"). $C$ can be a product space, to take into account several variables (fuel or battery gauge, accumulated fatigue, $\mathrm{CO}_{2}$ budget, available visas, etc.)

A contextual network on $X$ with context set $C$ is a network $N$ on the set of states $X \times C$, i.e. $N$ is a set endowed with two functions: $s, e: N \rightarrow X \times C$. Elements of $N$ are still called arcs: given an arc $\alpha$ we call $s(\alpha)$ and $e(\alpha)$ its starting state and endstate; we write $s_{X}, e_{X}: N \rightarrow X$ and $s_{C}, e_{C}: N \rightarrow C$ the functions defined by

$$
s(\alpha)=s_{X}(\alpha)^{s_{C}(\alpha)} \quad \text { and } \quad e(\alpha)=e_{X}(\alpha)^{e_{C}(\alpha)} \quad \forall \alpha \in N,
$$

in other words, $s_{X}(\alpha)$ and $e_{X}(\alpha)$ are the starting point and endpoint of the arc $\alpha$, while $s_{C}(\alpha)$ and $e_{C}(\alpha)$ are its starting and ending contexts. As before, several arcs can be used to describe trips corresponding to the same displacement in the physical space; actually, most of the time a lot of different arcs will be needed for each movement in the physical space to take into account the starting context. Even for a given movement and a given starting context, several arcs can be used, e.g. one for fast driving and another for slow driving (with different ending contexts, notably in term of fuel consumption and fatigue). The difference between $s_{C}(\alpha)$ and $e_{C}(\alpha)$ will model the change in context incurred by travelling along the $\operatorname{arc} \alpha$; the abundance of arcs with a certain starting context will model the variety of movements available given the context, so that our framework can be used to model e.g. cost constraints from the user perspective, see examples below.

Given a contextual network $N$ on a space $X$ with context $C$, a path $\gamma$ from $p^{c} \in X \times C$ to $q^{b} \in X \times C$ is a finite word $\alpha_{1} \alpha_{2} \ldots \alpha_{k}$ with letters in $N$ such that $s\left(\alpha_{1}\right)=p^{c}, e\left(\alpha_{k}\right)=q^{b}$ and $e\left(\alpha_{i}\right)=s\left(\alpha_{i+1}\right)$ for each $i \in\{1,2, \ldots, k-1\}$. We write $\gamma: p^{c} \leadsto q^{b}$ to express that $\gamma$ is some path from $p^{c}$ to $q^{b}$, but we may want to forget some information by speaking of a path from $p$ to $q$, writing $\gamma: p^{*} \sim q^{*}$; or of a path from $p^{c}$ to $q$ by writing $\gamma: p^{c} \leadsto q^{*}$. The set of paths is again denoted by $N^{*}$, and includes a trivial path $\varnothing_{p^{c}}: p^{c} \rightarrow p^{c}$ for each state $p^{c}$. Two paths $\gamma: p^{c} \leadsto q^{b}$ and $\eta: p^{\prime c^{\prime}} \leadsto q^{\prime b^{\prime}}$ are chainable if $p^{\prime c^{\prime}}=q^{b}$ (i.e. $p^{\prime}=q$ and $c^{\prime}=b$ ), and the concatenation of two chainable paths is a path denoted by $\gamma \cdot \eta$. 
A length function on a contextual network $N$ is a function $\ell: N \rightarrow[0,+\infty)$ such that $\ell(\alpha)>0$ whenever $s_{X}(\alpha) \neq e_{X}(\alpha)$ (we allow zero-cost arcs, as long as they do not entail movement but only change of context). We extend it to a function on $N^{*}$, still denoted by $\ell$, by

$$
\ell\left(\alpha_{1} \cdots \alpha_{k}\right)=\sum_{i=1}^{k} \ell\left(\alpha_{i}\right)
$$

with again the convention $\ell\left(\varnothing_{p^{c}}\right)=0$.

\section{A.4.2 Contextual distances}

Given a space $X$, a context set $C$, a contextual network $N$ and a length function $\ell$, we define an associated contextual quasimetric by

$$
d\left(p^{c}, q^{b}\right)=\inf _{\gamma: p^{c} \sim q^{b}} \ell(\gamma)
$$

This is not always a contextual metric, and as in the context-free case we introduce the following definition to ensure separation.

Definition A.10. We say that the contextual length function is non-degenerate when there exist a function $m:\{(p, q): p, q \in X, p \neq q\} \rightarrow(0,+\infty)$ such that for all $p \neq q \in X$, all $c, b \in C$ and all $\gamma: p^{*} \leadsto q^{*}$ we have $\ell(\gamma)>m(p, q)$.

With this at hand, we have the following neat relation between contextual networks and contextual metrics.

Theorem A.11. For all contextual data $(X, C, N, \ell)$ the associated contextual quasimetric has the Identity and Triangle Inequality properties. If in addition $\ell$ is nondegenerate, then $d$ has the Separation In Space property, and is thus a contextual metric.

Proof. The Identity property follows from the inclusion of empty paths $\varnothing_{p^{c}}$. The Triangle Inequality follows exactly as in Theorem A.5 from optimization. When $\ell$ is non-degenerate, we get immediately Separation In Space: whenever $p \neq q$, $\inf _{c, b} d\left(p^{c}, q^{b}\right)=\inf _{\gamma: p^{*} \sim q^{*}} \ell(\gamma) \geq m(p, q)>0$.

We can phrase the semi-specific and minimal distance functions and the maximal quasimetric in term of $N$ and $\ell$ consistently with their definition from $d$ :

$$
\begin{aligned}
d^{c, *}(p, q) & =\inf _{\gamma: p^{c} \sim q^{*}} \ell(\gamma) & d^{*, b}(p, q) & =\inf _{\gamma: p^{*} \sim q^{b}} \ell(\gamma) \\
d_{\min }(p, q) & =\inf _{\gamma: p^{*} \sim q^{*}} \ell(\gamma) & d_{\max }(p, q) & =\sup _{c} \inf _{\gamma: p^{c} \sim q^{*}} \ell(\gamma) .
\end{aligned}
$$




\section{Acknowledgements}

The authors thank T. Smith for encouraging them to pursue the idea of introducing a formula of distance that would care for the triangle inequality property, and editors and anonymous readers for helping in refining the reflection and expression on geographical distance.

This research is conducted under the umbrella of the project "Voyageur virtuel" funded by Université Gustave Eiffel.

\section{References}

[1] Veronique Van Acker, Bert Van Wee, and Frank Witlox. "When Transport Geography Meets Social Psychology: Toward a Conceptual Model of Travel Behaviour". In: Transport Reviews 30.2 (Mar. 2010), pp. 219-240. ISSN: 01441647. DOI: $10 / \operatorname{cf} 9 \mathrm{w} 7 \mathrm{c}$.

[2] Nobbir Ahmed and Harvey J. Miller. "Time-Space Transformations of Geographic Space for Exploring, Analyzing and Visualizing Transportation Systems". In: Journal of Transport Geography 15.1 (2007), pp. 2-17. ISSN: 09666923. DOI: $10 / \mathrm{dbgnd3.}$

[3] Shlomo Angel and Geoffrey Hyman. "Urban Fields". In: Pion Limited (1976). ISSN: 0850-860520.

[4] Luc Anselin and Xun Li. "Tobler's Law in a Multivariate World". en. In: Geographical Analysis n/a.n/a (2020). ISSN: 1538-4632. DOI: 10/ggw5xd.

[5] Afian Anwar, Wei Zeng, and Stefan Müller Arisona. "Time-Space Diagram Revisited". en. In: Transportation Research Record 2442.1 (2014), pp. 1-7. ISSN: 0361-1981. DOI: 10/ggw56w.

[6] Kay W. Axhausen et al. "Constructing Time-Scaled Maps: Switzerland from 1950 to 2000". In: Transport Reviews 28.3 (2008), pp. 391-413. DOI: 10/dvfqb2.

[7] Antoine Bailly. "Distances et Espaces: Vingt Ans de Géographie Des Représentations". In: L'espace géographique 3 (1985), pp. 197-205. DOI: 10/ggw564.

[8] Hannah Bast et al. "Route Planning in Transportation Networks". en. In: Algorithm Engineering: Selected Results and Surveys. Ed. by Lasse Kliemann and Peter Sanders. Lecture Notes in Computer Science. Cham: Springer International Publishing, 2016, pp. 19-80. ISBN: 978-3-319-49487-6. DOI: 10/dxpf.

[9] Jean-Jacques Bavoux. "Structuration du territoire bourguignon : de l'île à l'isthme". fr. In: Revue Géographique de l'Est 46.1-2 (2009). ISSN: 035-3213. 
[10] H. Beguin and Jacques François Thisse. "An Axiomatic Approach to Geographical Space". In: Geographical Analysis 11.4 (1979), pp. 325-341. DOI: 10/d4qrw5.

[11] J.M. Blaut. "Space and Process". In: Professional geographer 13.4 (1961), pp. 1-7. DOI: 10/fcxkt7.

[12] William Bunge. Theoretical Geography. seconde éd. augmentée 1966. Lund: Gleerup, 1962.

[13] L. G. Chalmet, R. L. Francis, and P. B. Saunders. "Network Models for Building Evacuation”. en. In: Fire Technology 18.1 (Feb. 1982), pp. 90-113. ISSN: 15728099. DOI: 10/b3qtxk.

[14] Laurent Chapelon. "L'accessibilité, marqueur des inégalités de rayonnement des villes portuaires en Europe". fr. In: Cybergeo : European Journal of Geography (2006). ISSN: 1278-3366. DOI: 10/c95s4b.

[15] Walter Collischonn and Jorge Victor Pilar. "A Direction Dependent LeastCost-Path Algorithm for Roads and Canals". In: International Journal of Geographical Information Science 14.4 (2000), pp. 397-406. DOI: 10/bg5jgj.

[16] Helen Couclelis. "Space, Time, Geography". In: Geographical Information Systems: Principles, Techniques, Management, and Applications. Wiley. Vol. 1. 1999, pp. 29-38.

[17] Henri Paul de Saint-Gervais. Uniformization of Riemann Surfaces. 2016. ISBN: 978-3-03719-145-3. DOI: 10/dxph.

[18] D J Dean. "Finding Optimal Routes for Networks of Harvest Site Access Roads Using GIS-Based Techniques". In: Canadian Journal of Forest Research 27.1 (1997), pp. 11-22. ISSN: 0045-5067. DOI: 10/cmvc46.

[19] Matthias Delafontaine, Tijs Neutens, and Nico Van de Weghe. "A GIS Toolkit for Measuring and Mapping Space-Time Accessibility from a Place-Based Perspective". In: International Journal of Geographical Information Science 26.6 (2012), pp. 1131-1154. ISSN: 1365-8816. DOI: 10/ggw56x.

[20] Daniel Delling et al. "Engineering Route Planning Algorithms". In: Algorithmics of Large and Complex Networks. Springer, 2009, pp. 117-139. ISBN: 9783-642-02094-0.

[21] Tamás Dusek and Gábor Szalkai. "Time- Spaces in Hungary". In: Raportul spaţiu-timp în Ungaria. 16.2 (2017), pp. 161-170. ISSN: 15831523. DOI: $10 /$ ggw57q. 
[22] Péter Ficzere, Zita Ultmann, and Ádám Török. "Time-Space Analysis of Transport System Using Different Mapping Methods". In: Transport ahead-of-print (2014), pp. 1-7. DOI: 10/ggw57s.

[23] Anthony C. Gatrell. Distance and Space: A Geographical Perspective. Clarendon Press Oxford, 1983.

[24] Stan C. M. Geertman and Jan R. Ritsema Van Eck. "GIS and Models of Accessibility Potential: An Application in Planning". In: International Journal of Geographical Information Systems 9.1 (1995), pp. 67-80. ISSN: 0269-3798. DOI: $10 /$ dxkxn4.

[25] Reginald George Golledge. Spatial Behavior: A Geographical Perspective. en. Guilford Press, 1997. ISBN: 1-57230-050-7.

[26] Torsten Hägerstrand. "What about People in Regional Science?" In: Papers of the Regional Science Association 24 (1970), pp. 7-21. DOI: 10/bkqqfc.

[27] Peter Haggett. Geography, a Global Synthesis. Harlow: Prentice Hall, 2001.

[28] Randolph W. Hall. "The Fastest Path through a Network with Random TimeDependent Travel Times". In: Transportation science 20.3 (1986), pp. 182-188. DOI: $10 / \mathrm{c56gbh}$.

[29] Jean-Marie Huriot, Tony E. Smith, and Jacques François Thisse. "MinimumCost Distances in Spatial Analysis". en. In: Geographical Analysis 21.4 (1989), pp. 294-315. ISSN: 1538-4632. DOI: 10/bxd4s2.

[30] G.M. Hyman and L. Mayhew. "Advances in Travel Geometry and Urban Modelling". In: GeoJournal 59 (2004), pp. 191-207. DOI: 10/cjpm42.

[31] Hildebert Isnard et al. Problématiques de la géographie. français. SUP, Le géographe. Paris: Presses universitaires de France, 1981. ISBN: 2-13-036800-X.

[32] H.-M. Kim and M.-P. Kwan. "Space-Time Accessibility Measures: A Geocomputational Algorithm with a Focus on the Feasible Opportunity Set and Possible Activity Duration". In: Journal of Geographical Systems 5.1 (2003), pp. 7191. DOI: 10/cghvmc.

[33] S. Kirsch. "The Incredible Shrinking World? Technology and the Production of Space". In: Environment and Planning D: Society and Space 13.5 (1995), pp. 529-555. DOI: 10/bq4fzn.

[34] Menno-Jan Kraak. "The Space-Time Cube Revisited from a Geovisualization Perspective". In: Proc. 21st International Cartographic Conference. Durban: Citeseer, 2003, pp. 1988-1996. 
[35] J. Kruskal and M. Wish. Multidimensional Scaling. Beverly Hills/London: Sage Publications, 1978.

[36] Bart Kuijpers, Harvey J. Miller, Tijs Neutens, et al. "Anchor Uncertainty and Space-Time Prisms on Road Networks". In: International Journal of Geographical Information Science 24.8 (June 2010), pp. 1223-1248. ISSN: 1365-8816. DOI: $10.1080 / 13658810903321339$.

[37] Bart Kuijpers, Harvey J. Miller, and Walied Othman. "Kinetic Prisms: Incorporating Acceleration Limits into Space-Time Prisms". In: International Journal of Geographical Information Science 31.11 (2017), pp. 2164-2194. ISSN: 13658816. DOI: 10/ggw56v.

[38] Alain L'Hostis. "All Geographical Distances Are Optimal". en. In: Cybergeo : European Journal of Geography (June 2020). ISSN: 1278-3366. DOI: 10/gg3rbx.

[39] Alain L'Hostis. "Detour and Break Optimising Distance, a New Perspective on Transport and Urbanism". In: Environment and Planning B: Planning and Design 44.3 (2017), pp. 441-463. DOI: 10/gddwqw.

[40] Alain L'Hostis. "Misunderstanding Geographical Distances: Two Errors and an Issue in the Interpretation of Violations of the Triangle Inequality". In: Cybergeo : European Journal of Geography 793 (2016). DOI: 10/ggw58h.

[41] Alain L'Hostis. "The Shrivelled USA: Representing Time-Space in the Context of Metropolitanization and the Development of High-Speed Transport". In: Journal of Transport Geography 17.6 (2009), pp. 433-439. ISSN: 0966-6923. DOI: $10 /$ dv69r3.

[42] T. L. Lei and R. L. Church. "Mapping Transit-based Access: Integrating GIS, Routes and Schedules". In: International Journal of Geographical Information Science 24.2 (2010), pp. 283-304. ISSN: 1365-8816. DOI: 10/cqq6zd.

[43] Jacques Lévy. "Entre Contact et Écart: La Distance Au Coeur de La Réflexion". In: Atala 12 (2009), pp. 175-185.

[44] A. Lozano and G. Storchi. "Shortest Viable Path Algorithm in Multimodal Networks". In: Transportation Research Part A: Policy and Practice 35.3 (2001), pp. 225-241. DOI: 10/bptsq9.

[45] Tai-Yu Ma and Jean-Patrick Lebacque. "Dynamic System Optimal Routing in Multimodal Transit Network". en. In: Transportation Research Record 2351.1 (Jan. 2013), pp. 76-84. ISSN: 0361-1981. DOI: 10/ggw58v. 
[46] Bernard Marchand. "Deformation of a Transportation Surface". In: Annals of the Association of American Geographers 63 (1973), pp. 507-521. DOI: $10 /$ dj9dz9.

[47] David M. Mark et al. "Cognitive Models of Geographical Space". In: International Journal of Geographical Information Science 13.8 (Dec. 1999), pp. 747774. ISSN: 1365-8816. DOI: 10/fsgj2d.

[48] Philippe Mathis. "Espace et graphe, le p-graphe t-modal 1-planaire". FR. In: Table ronde ASRDLF "Distance et analyse spatiale". Chamonix: ASRDLF, 1990, p. 10.

[49] Philippe Mathis, Nadine Polombo, and Alain L'Hostis. "Les Grandes Vitesses". In: Circuler Demain. Monde En Cours, Prospective et Territoires. La Tour d'Aigues: DATAR Editions de l'Aube, 1993, pp. 129-142.

[50] Peter Merriman. "Human Geography without Time-Space". en. In: Transactions of the Institute of British Geographers 37.1 (2012), pp. 13-27. ISSN: 14755661. DOI: $10 /$ drnx8c.

[51] Harvey J. Miller. "A Measurement Theory for Time Geography". en. In: Geographical Analysis 37.1 (2005), pp. 17-45. ISSN: 1538-4632. DOI: 10/fsbx9f.

[52] Harvey J. Miller. "Activities in Space and Time". In: Handbook of Transport 5: Transport Geography and Spatial Systems. Pergamon/Elsevier Science, 2004.

[53] Harvey J. Miller. "Tobler's First Law and Spatial Analysis". In: Annals of the Association of American Geographers 94.2 (2004), pp. 284-289. DOI: $10 /$ dh39xr.

[54] Harvey J. Miller and Elizabeth A. Wentz. "Representation and Spatial Analysis in Geographic Information Systems". In: Annals of the Association of American Geographers 93.3 (2003), pp. 574-594. DOI: 10/c32mk3.

[55] Patricia L. Mokhtarian and Ilan Salomon. "How Derived Is the Demand for Travel? Some Conceptual and Measurement Considerations". In: Transportation research part A: Policy and practice 35.8 (2001), pp. 695-719. DOI: $10 /$ bs $8 \mathrm{vkm}$.

[56] Daniel R. Montello. "The Measurement of Cognitive Distance: Methods and Construct Validity". In: Journal of Environmental Psychology 11.2 (1991), pp. 101-122. DOI: 10/dcmpf9. 
[57] Matthias Müller-Hannemann et al. "Timetable Information: Models and Algorithms". en. In: Algorithmic Methods for Railway Optimization. Ed. by Frank Geraets et al. Lecture Notes in Computer Science. Springer Berlin Heidelberg, 2007, pp. 67-90. ISBN: 978-3-540-74247-0.

[58] A. Nuzzolo, F. Russo, and U. Crisalli. "A Doubly Dynamic Schedule-Based Assignment Model for Transit Networks". In: Transportation Science 35.3 (2001), pp. 268-285. DOI: 10/c485gr.

[59] Morton E O'Kelly. "Equilibrium in a Two-Crop Model with a Low-Cost Transportation Route". en. In: Environment and Planning A: Economy and Space 21.3 (Mar. 1989), pp. 385-396. ISSN: 0308-518X. DOI: 10.1068/a210385.

[60] David O'Sullivan, Alastair Morrison, and John Shearer. "Using Desktop GIS for the Investigation of Accessibility by Public Transport: An Isochrone Approach". In: International Journal of Geographical Information Science 14.1 (2000), pp. 85-104. ISSN: 1365-8816. DOI: 10/dx3sb7.

[61] François Plassard and Jean-Louis Routhier. Sémiologie Graphique et Évaluation. Lyon: A.R.T.U.R., 1987.

[62] Henri Poincaré. Science et méthode. FR. Flammarion, 1908.

[63] J. R Ritsema van Eck and T de Jong. "Accessibility Analysis and Spatial Competition Effects in the Context of GIS-Supported Service Location Planning". en. In: Computers, Environment and Urban Systems 23.2 (1999), pp. 75-89. ISSN: 0198-9715. DOI: 10/fgbkrh.

[64] Shih-Lung Shaw and Hongbo Yu. "A GIS-Based Time-Geographic Approach of Studying Individual Activities and Interactions in a Hybrid Physical-Virtual Space". In: Journal of Transport Geography. ICT and the Shaping of Access, Mobility and Everyday Life 17.2 (2009), pp. 141-149. ISSN: 0966-6923. DOI: $10 /$ cfnc 45 .

[65] R.N. Shepard. "The Analysis of Proximities: Multidimensional Scaling with an Unknown Distance Function". In: Psychometrika 27.2 (1962), pp. 125-140. DOI: $10 /$ cv5q67.

[66] Eihan Shimizu and Ryo Inoue. "A New Algorithm for Distance Cartogram Construction". In: International Journal of Geographical Information Science 23.11 (2009), pp. 1453-1470. ISSN: 13658816. DOI: 10/bbfq5r.

[67] Tony E. Smith. "Shortest-Path Distances: An Axiomatic Approach". In: Geographical analysis 21.1 (1989), pp. 1-31. DOI: 10/dnj2wj. 
[68] Daniel Z. Sui. “Tobler's First Law of Geography: A Big Idea for a Small World?” In: Annals of the Association of American Geographers 94.2 (2004), pp. 269277. DOI: $10 / \mathrm{fk} 249 \mathrm{f}$.

[69] Waldo Rudolph Tobler. "A Computer Movie Simulating Urban Growth in the Detroit Region”. In: Economic geography 46 (1970), pp. 234-240. DOI: 10 / cfqcnv.

[70] Waldo Rudolph Tobler. Three Presentations on Geographical Analysis and Modeling: Non- Isotropic Geographic Modeling; Speculations on the Geometry of Geography; and Global Spatial Analysis. Tech. rep. 93-1. NCGIA, 1993, p. 24.

[71] Waldo Rudolph Tobler. "Visualizing the Impact of Transportation on Spatial Relations". In: Western Regional Science Association Meeting. Hawaii: Western Regional Science Association, 1997, p. 7.

[72] Wei Tu et al. "Optimizing the Locations of Electric Taxi Charging Stations: A Spatial-Temporal Demand Coverage Approach". en. In: Transportation Research Part C: Emerging Technologies 65 (Apr. 2016), pp. 172-189. ISSN: 0968090X. DOI: $10 /$ f8d6dg.

[73] William Warntz. "Global Science and the Tyranny of Space". en. In: Papers of the Regional Science Association 19.1 (1967), pp. 6-19. ISSN: 1435-5957. DOI: $10 / \mathrm{dz} 96 \mathrm{kp}$.

[74] Devin A. White and Sarah B. Barber. "Geospatial Modeling of Pedestrian Transportation Networks: A Case Study from Precolumbian Oaxaca, Mexico". In: Journal of archaeological science 39.8 (2012), pp. 2684-2696. DOI: $10 /$ gd6b24.

[75] Tim Williams. The Silk Roads: An ICOMOS Thematic Study. EN. Monographie. UNESCO WHC, 2014, p. 152.

[76] Wallace Alvin Wilson. "A Relation Between Metric and Euclidean Spaces". In: American Journal of Mathematics 54.3 (1932), pp. 505-517. ISSN: 0002-9327. DOI: 10/c97ztp.

[77] Wallace Alvin Wilson. "On Semi-Metric Spaces". In: American Journal of Mathematics 53.2 (1931), pp. 361-373. ISSN: 0002-9327. DOI: 10/cnp23h.

[78] Yi-Hwa Wu and Harvey J. Miller. "Computational Tools for Measuring SpaceTime Accessibility within Dynamic Flow Transportation Networks". In: Journal of Transportation and Statistics 4.2/3 (2001), pp. 1-14. 
[79] M. Yuan. "Space-Time Modeling". In: International Encyclopedia of Human Geography. Oxford: Elsevier, 2009, pp. 286-295. ISBN: 978-0-08-044910-4.

[80] Yacov Zahavi. Travel Characteristics in Cities of Developing and Developed Countries. Staff Working Paper 230. International Bank for Reconstruction and Development, 1976, p. 113.

[81] W. Zeng et al. "Visualizing Mobility of Public Transportation System". In: IEEE Transactions on Visualization and Computer Graphics 20.12 (2014), pp. 1833-1842. ISSN: 1077-2626. DOI: 10/f6qjqw.

[82] George Kingsley Zipf. Human Behaviour and the Principle of Least-Effort. Cambridge, USA: Addison-Wesley, 1949. 